\title{
Subgap features due to quasiparticle tunneling in quantum dots coupled to superconducting leads
}

\author{
Sebastian Pfaller, Andrea Donarini, and Milena Grifoni \\ Theoretische Physik, Universität Regensburg, 93040 Regensburg, Germany \\ (Received 30 August 2012; revised manuscript received 12 February 2013; published 30 April 2013)
}

\begin{abstract}
We present a microscopic theory of transport through quantum dot setups coupled to superconducting leads. We derive a master equation for the reduced density matrix to lowest order in the tunneling Hamiltonian and focus on quasiparticle tunneling. For high enough temperatures transport occurs in the subgap region due to thermally excited quasiparticles, which can be used to observe excited states of the system at low bias voltages. On the example of a double quantum dot we show how subgap transport spectroscopy can be done. Moreover, we use the single level quantum dot coupled to a normal and a superconducting lead to give a possible explanation for the subgap features observed in the experiments of Dirks, Chen, Birge, and Mason [Appl. Phys. Lett. 95, 192103 (2009)].
\end{abstract}

DOI: 10.1103/PhysRevB.87.155439

PACS number(s): 73.23.Hk, 73.63.Kv, 74.45.+c

\section{INTRODUCTION}

In the last two decades modern fabrication techniques made it possible to connect quantum dot systems with superconducting leads. Quantum dots were realized with carbon nanotubes, ${ }^{1-7}$ metallic particles, ${ }^{8}$ semiconducting nanowires, ${ }^{9-12}$ single fullerene molecules, ${ }^{13}$ self-assembled nanocrystals, ${ }^{14}$ and graphene quantum dots. ${ }^{15}$ The experiments show a gap in the Coulomb diamonds which is proportional to the superconducting gap, reflecting the BCS density of states. In the sequential tunneling regime higher order quasiparticle tunneling processes are suppressed and current flows due to single quasiparticle tunneling. First transport theories were presented, ${ }^{16}$ using a master equation approach, where the rates were calculated on the basis of Fermi's golden rule. Another method based on nonequilibrium Green's function was used by Yeyati et al. ${ }^{17}$ and $\mathrm{Kang}^{18}$ to describe resonant tunneling through an effective single level quantum dot in the limit of very strong Coulomb repulsion in the $\operatorname{dot}(U \rightarrow \infty$ limit), where transport is governed by quasiparticle tunneling; the corresponding $I-V$ curves show an intrinsic broadening of the BCS-like feature in the current in agreement with experimental observation. ${ }^{8}$ For small Coulomb repulsion, higher order processes lead to Josephson current ${ }^{9}$ and Andreev reflections, ${ }^{2-5,7,10,15}$ which appear as subgap features in the experiments. Both effects were studied intensely experimentally and theoretically $4,17,19,20$ and were recently summarized in review articles of Refs. 21 and 22. Besides Andreev reflections, also the Kondo effect ${ }^{13}$ as well as Yu-Shiba-Rusinov bound states ${ }^{5,23,24}$ can lead to subgap features and are the subject of current research. If the temperature becomes comparable with the superconducting gap quasiparticles can get thermally excited across the gap, leading to additional subgap features. ${ }^{16}$

In the following we present a microscopic theory for transport through superconducting hybrid nanojunctions for finite superconducting gap $|\Delta|<\infty$ in the sequential tunneling limit. In particular, we trace out all degrees of freedom of the superconducting leads to obtain a generalized master equation for the reduced density matrix to lowest order in the tunneling Hamiltonian. We differentiate from Ref. 16 by going beyond the constant interaction implicitly used there, and from Refs. 17 and 18 since we also treat subgap features associated to many-body excitations of a quantum dot molecule (double quantum dot). In contrast to Green's function techniques (see e.g., Ref. 22), this method enables one to treat the interactions on the system exactly. Moreover, as shown in the example of a double quantum dot, our theory is easily scalable and allows an exact treatment of the Coulomb interaction and can treat any quantum dot setup. Hence, we can describe lowest order quasiparticle transport of experimental relevant quantum dot systems (multiple quantum dots or multilevel quantum dots). We focus on transport involving thermally excited quasiparticles, and show that excited states of the quantum dot system can be observed in the current-voltage spectroscopy in the Coulomb blockade region. Though transitions between two ground states are blocked due to the gap in the BCS density of states, thermally excited quasiparticles can participate in transport through excited system states, giving a source of subgap features in superconducting hybrid systems. These subgap features are already present in lowest order of the perturbation theory, in contrast to Cooper pair transport which occurs only in fourth order in the tunneling coupling. Nevertheless, experiments suggest the existence of a regime in which quasiparticle transport dominates also in the subgap region. ${ }^{21}$ For a quantum dot coupled to a normal and a superconducting lead, a possible explanation for the subgap features observed in Ref. 1 is given, where a carbon nanotube quantum dot is coupled to a normal and a superconducting contact.

The paper is organized as follows: In Sec. II we introduce the Hamiltonian in a system-bath model using a number conserving version of the Bogoliubov-Valatin transformation. ${ }^{25,26}$ We describe the electrons of the superconducting leads as a combination of quasiparticle excitations of the BCS ground state and Cooper pairs. For this purpose we introduce Cooper pair creation and annihilation operators. The explicit inclusion of these operators allows one to construct a theory which conserves the particle number in the tunneling process. In this way, for example, anomalous contributions to the tunneling rates due to Cooper pairing naturally vanish in second order. In Sec. III, the generalized master equation for the reduced density matrix is derived and used to calculate the current. In Sec. IV we apply the theory to the calculation of transport characteristics of two systems: the single level quantum dot 

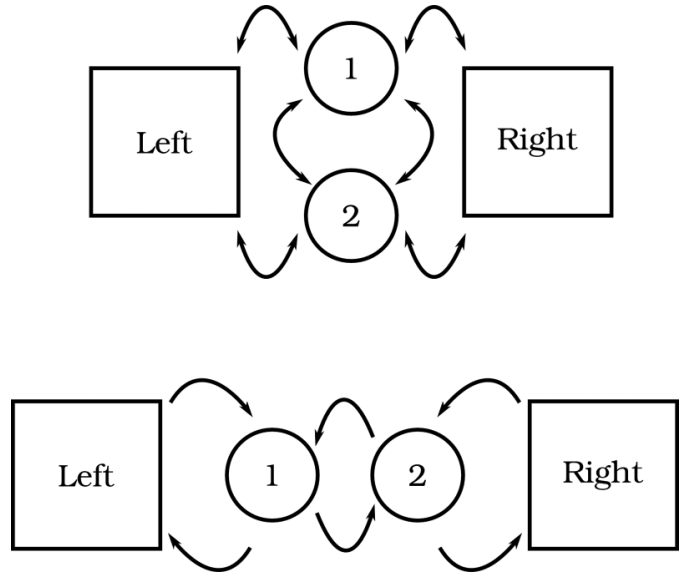

FIG. 1. Sketch of the transport setup of a DD coupled to superconducting leads. The DD is illustrated in the parallel (top panel) and serial (bottom panel) configurations. Tunneling events are depicted by arrows.

(SD) and the double quantum dot (DD), the latter in two possible configurations (cf. Fig. 1). The SD is used to explain basic phenomena such as a gap opening in the Coulomb diamonds which is proportional to the superconducting gap, and transport involving thermally excited quasiparticles. ${ }^{16} \mathrm{On}$ the other hand, the DD possess a richer many-body spectrum with several excited states. We visualize transitions through excited system states in the low bias regime using thermally excited quasiparticles. Due to the gap in the BCS density of states, the ground state to ground state transition is not allowed in all cases, leading to transport through excited system states, appearing as peaks in the Coulomb blockade region. The threshold for observing excited system states in the subgap region is that the energy difference between the excited state and its ground state must be smaller than $2|\Delta|$. We confirmed this threshold by means of the independently gated $\mathrm{DD}$, where the detuning of the two sites changes the level spacing. Finally the N-QD-S system is investigated, where a quantum dot is coupled to a normal and a superconducting lead. In this case only the superconducting lead produces thermal lines in the Coulomb blockade region, giving a possible explanation for the subgap features in Ref. 1.

\section{MODEL HAMILTONIAN}

In the following we consider quantum dot systems weakly coupled to two superconducting leads. The total Hamiltonian is written in a system-bath model:

$$
\hat{H}=\hat{H}_{S}+\hat{H}_{B}+\hat{H}_{T},
$$

where $\hat{H}_{S}$ represents the Hamiltonian of the quantum dot system, $\hat{H}_{B}$ is the Hamiltonian of the superconducting leads, and $\hat{H}_{T}$ describes the tunneling between the system and the leads. Specifically, we focus on two systems: a single level quantum dot (SD) and a double quantum dot (DD). The SD has been the focus of many theoretical works before,,${ }^{16-20}$ and we use its simple Fock-space structure to demonstrate some generic effects resulting from the superconducting leads.
We describe the SD by the single impurity Anderson model:

$$
\hat{H}_{\mathrm{SD}}=\sum_{\sigma} \epsilon_{d} \hat{d}_{\sigma}^{\dagger} \hat{d}_{\sigma}+U \hat{n}_{\uparrow} \hat{n}_{\downarrow},
$$

where $\hat{n}_{\sigma}=\hat{d}_{\sigma}^{\dagger} \hat{d}_{\sigma}$ is the number operator of the electrons on the dot with spin $\sigma$. This model describes a quantum dot with on-site energy $\epsilon_{d}$ and Coulomb repulsion $U$ which can be occupied by at most two electrons. The highest occupied state is defined as $|2\rangle=\hat{d}_{\uparrow}^{\dagger} \hat{d}_{\downarrow}^{\dagger}|0\rangle$, the 1-particle states are defined as $|1 \sigma\rangle=\hat{d}_{\sigma}^{\dagger}|0\rangle$, and $|0\rangle$ is the state with zero particles.

For the DD we use a modified version of the Pariser-ParrPople Hamiltonian: ${ }^{27,28}$

$$
\begin{aligned}
\hat{H}_{\mathrm{DD}}= & \sum_{\substack{\alpha \in\{1,2\} \\
\sigma \in\{\uparrow, \downarrow\}}} \epsilon_{\alpha \sigma} \hat{d}_{\alpha \sigma}^{\dagger} \hat{d}_{\alpha \sigma}+\sum_{\sigma}\left(b \hat{d}_{1 \sigma}^{\dagger} \hat{d}_{2 \sigma}+b^{*} \hat{d}_{2 \sigma}^{\dagger} \hat{d}_{1 \sigma}\right) \\
& +\sum_{\alpha} U_{\alpha}\left(\hat{n}_{\alpha \uparrow}-\frac{1}{2}\right)\left(\hat{n}_{\alpha \downarrow}-\frac{1}{2}\right) \\
& +V\left(\hat{n}_{1}-1\right)\left(\hat{n}_{2}-1\right) .
\end{aligned}
$$

Here, $\hat{d}_{\alpha \sigma}^{\dagger}$ are the creation operators for an electron on site $\alpha \in$ $\{1,2\}$ with spin $\sigma$. They define the number operators $\hat{n}_{\alpha \sigma}=$ $\hat{d}_{\alpha \sigma}^{\dagger} \hat{d}_{\alpha \sigma}$. The operator $\hat{n}_{\alpha}=\hat{n}_{\alpha \uparrow}+\hat{n}_{\alpha \downarrow}$ counts the number of electrons on site $\alpha$. In the general case we distinguish between the four on-site energies $\epsilon_{\alpha \sigma}$ and between the on-site Coulomb interactions $U_{\alpha}$. Electrons on different sites interact through the interdot Coulomb interaction $V ; b$ describes the hopping between the two sites. In our setup the on-site energies can be controlled by capacitively coupled gate electrodes. In the case of site-independent on-site energies and on-site Coulomb interaction the Hamiltonian can be diagonalized analytically. ${ }^{29,30}$

The superconducting leads are described by the mean-field form $\hat{H}_{B}^{\mathrm{MF}}$ of the pairing Hamiltonian, where we additionally inserted a unity represented by a product of Cooper pair annihilation and creation operators, $\hat{S}_{\eta} \hat{S}_{\eta}^{\dagger}=1$, which will be specified later in Sec. II. We find

$$
\begin{aligned}
\hat{H}_{B}^{\mathrm{MF}}= & \sum_{\eta k \sigma} \xi_{\eta k} \hat{c}_{\eta k \sigma}^{\dagger} \hat{c}_{\eta k \sigma}+\sum_{\eta} \mu_{\eta} \hat{N}_{\eta} \\
& +\sum_{\eta k}\left(\Delta_{\eta} \hat{c}_{\eta k \uparrow}^{\dagger} \hat{c}_{\eta-k \downarrow}^{\dagger} \hat{S}_{\eta}+\Delta_{\eta}^{*} \hat{S}_{\eta}^{\dagger} \hat{c}_{\eta-k \downarrow} \hat{c}_{\eta k \uparrow}\right) \\
= & \hat{H}_{G}+\sum_{\eta} \mu_{\eta} \hat{N}_{\eta},
\end{aligned}
$$

where $\xi_{\eta k}=\epsilon_{k}-\mu_{\eta}$ measures single-particle energies $\epsilon_{k}$ with respect to the electrochemical potential $\mu_{\eta}$, and $\hat{N}_{\eta}=$ $\sum_{k \sigma} \hat{c}_{\eta k \sigma}^{\dagger} \hat{c}_{\eta k \sigma}$ counts the number of electrons in lead $\eta$. Finally, $\Delta_{\eta}=\left|\Delta_{\eta}\right| e^{i \phi_{\eta}} \equiv-\sum_{l} V_{l k}\left\langle\hat{S}_{\eta}^{\dagger} \hat{c}_{\eta-k \downarrow} \hat{c}_{\eta k \uparrow}\right\rangle$ denotes the superconducting gap of lead $\eta$. Here $\langle\cdot\rangle$ denotes a thermal average calculated self-consistently using the mean-field Hamiltonian of Eq. (4).

The tunneling Hamiltonian

$$
\hat{H}_{T}=\sum_{\eta k \sigma \alpha} t_{\eta \alpha \sigma} \hat{c}_{\eta k \sigma}^{\dagger} \hat{d}_{\alpha \sigma}+t_{\eta \alpha \sigma}^{*} \hat{d}_{\alpha \sigma}^{\dagger} \hat{c}_{\eta k \sigma}
$$

describes the tunneling between the leads and the two sites of the DD, where the tunneling coefficients $t_{\eta \alpha \sigma}$ depend on 
the lead, site, and spin index. Depending on the choice of the tunneling coefficients the DD is described in parallel or in serial configuration (see Fig. 1). For the single dot we skip the index $\alpha$ in Eq. (5), as only one site is involved.

\section{A. Diagonalization of the lead Hamiltonian}

The most famous way to diagonalize the mean-field Hamiltonian $\hat{H}_{B}^{\mathrm{MF}}$ of Eq. (4) was first introduced by Bogoliubov. ${ }^{31}$ We are following Josephson and Bardeen ${ }^{25,26}$ who modified the so-called Bogoliubov transformation in a number conserving way. We adopt this idea and define the Bogoliubov transformation:

$$
\hat{c}_{\eta k \sigma}^{\dagger}=u_{\eta k} \hat{\gamma}_{\eta k \sigma}^{\dagger}+\operatorname{sgn} \sigma v_{\eta k}^{*} \hat{\gamma}_{\eta-k \sigma} \hat{S}_{\eta}^{\dagger},
$$

where $\bar{\sigma}=-\sigma$. In Eq. (6) $\hat{\gamma}_{\eta k \sigma}^{\dagger}$ creates a fermionic quasiparticle, often called bogoliubon, which is defined by

$$
\begin{gathered}
\left\{\hat{\gamma}_{\eta k \sigma}^{\dagger}, \hat{\gamma}_{\eta^{\prime} k^{\prime} \sigma^{\prime}}\right\}=\delta_{\eta \eta^{\prime}} \delta_{k k^{\prime}} \delta_{\sigma \sigma^{\prime}}, \\
\hat{\gamma}_{\eta k \sigma}|\mathrm{GS}\rangle_{\eta}=0 .
\end{gathered}
$$

Here $|\mathrm{GS}\rangle_{\eta}$ denotes the ground state, or Cooper pair condensate of lead $\eta{ }^{32}$ Bogoliubons are quasiparticle excitations of the Cooper pair condensate, meaning that the Cooper pair condensate is defined as the vacuum state of the bogoliubons [see Eq. (8)]. The coefficients $u_{\eta k}$ and $v_{\eta k}$ are complex numbers and fulfill

$$
\left|u_{\eta k}\right|^{2}+\left|v_{\eta k}\right|^{2}=1
$$

They read

$$
\begin{gathered}
u_{\eta_{k}}=\sqrt{\frac{1}{2}\left(1+\frac{\xi_{\eta k}}{\left|E_{\eta k}\right|}\right)}, \\
v_{\eta k}=e^{i \phi_{\eta}} \sqrt{\frac{1}{2}\left(1-\frac{\xi_{\eta k}}{\left|E_{\eta k}\right|}\right)},
\end{gathered}
$$

where $\phi_{\eta}$ is the phase of the superconducting gap $\Delta_{\eta}$.

In the number conserving description, the Hamiltonian of Eq. (4) commutes with the particle number operator. Hence, it is required that the ground state must be an eigenstate of the particle number operator. We define the ground state of lead $\eta$ as $^{33,34}|\mathrm{GS}\rangle_{\eta}=|0, N\rangle_{\eta}$, where $|0, N\rangle_{\eta}$ represents a state with $N / 2$ Cooper pairs and zero quasiparticle excitations. The Cooper pair annihilation operator $\hat{S}_{\eta}$ annihilates a Cooper pair in lead $\eta$ and can formally be defined as ${ }^{33}$

$$
\begin{aligned}
\hat{S}_{\eta}|0, N\rangle_{\eta} & =|0, N-2\rangle_{\eta}, \\
\hat{S}_{\eta}|k \sigma, N\rangle_{\eta} & =|k \sigma, N-2\rangle_{\eta}, \\
\hat{\gamma}_{k \sigma}^{\dagger}|0, N\rangle_{\eta} & =|k \sigma, N\rangle_{\eta} .
\end{aligned}
$$

Equation (12) implies that the Cooper pairs and the quasiparticles are decoupled:

$$
\left[\hat{S}_{\eta}^{\dagger}, \hat{\gamma}_{k \sigma}^{\dagger}\right]=0, \quad\left[\hat{S}, \hat{\gamma}_{k \sigma}^{\dagger}\right]=0
$$

and the Cooper pair operators have the following properties (see Appendix A):

$$
\hat{S}_{\eta} \hat{S}_{\eta}^{\dagger}=1, \quad\left[\hat{S}_{\eta}, \hat{S}_{\eta}^{\dagger}\right]=\hat{\mathcal{P}}_{0, \eta},
$$

where $\hat{\mathcal{P}}_{0}$ is the projector on states with zero Cooper pairs, and

$$
\left[\hat{N}, \hat{S}^{\dagger}\right]=2 \hat{S}^{\dagger} \text {. }
$$

Note that the transformation defined in Eq. (6) conserves the fermionic properties of the electron operators only if we restrict our Hilbert space to a subspace with more than zero Cooper pairs. In that subspace $\hat{S}$ commutes with $\hat{S}^{\dagger}$ and the Bogoliubov transformation is well defined.

Applying the transformation of Eq. (6) on Eq. (4) we obtain that

$$
\hat{H}_{B}-\sum_{\eta} \mu_{\eta} \hat{N}_{\eta}=\sum_{\eta k \sigma} E_{\eta k} \hat{\gamma}_{\eta k \sigma}^{\dagger} \hat{\gamma}_{\eta k \sigma}+E_{G}+T\left(\hat{\mathcal{P}}_{0}\right),
$$

where $T\left(\hat{\mathcal{P}}_{0}\right)$ are terms proportional to $\hat{\mathcal{P}}_{0}$. They vanish after truncating the Hilbert space and only diagonal contributions remain. In Eq. (16) $E_{\eta k}=\sqrt{\xi_{\eta k}^{2}+\left|\Delta_{\eta}\right|^{2}}$ denotes the quasiparticle energy, and $E_{G}$ is a constant energy offset, often referred to as the energy of the Cooper pair condensate. For later reference we note that the term $\sum_{\eta} \mu_{\eta} \hat{N}_{\eta}$ is not included in the diagonalization procedure and is still written in terms of electron operators.

\section{TRANSPORT THEORY AND THE GENERALIZED MASTER EQUATION}

In this section we derive the generalized master equation in the presence of superconducting leads. Since the generalized master equation approach to transport through quantum dots has become rather standard in recent years (see, e.g., the method article by Timm et al. ${ }^{35}$ or the recent paper by Koller et $a .^{36}{ }^{36}$, we only go into details of the derivation of the master equation when the effect of the superconducting leads brings significant differences with respect to the normal conducting theory.

The expectation value $O=\langle\hat{O}\rangle=\operatorname{Tr}(\hat{O} \hat{\rho})$ of any observable associated to an operator $\hat{O}$ can be evaluated once the total density operator $\hat{\rho}$ is known [cf. Eq. (34) below]. To this extent we start from the Liouville equation for the density operator in the interaction picture; see, e.g., ${ }^{37}$

$$
i \hbar \frac{\partial}{\partial t} \hat{\rho}_{I}(t)=\left[\hat{H}_{T, I}(t), \hat{\rho}_{I}(t)\right] .
$$

Equation (17) can be formally integrated and reinserted back into itself,

$$
\begin{aligned}
i \hbar \dot{\hat{\rho}}_{I}(t)= & {\left[\hat{H}_{T, I}(t), \hat{\rho}_{I}(0)\right] } \\
& -\frac{i}{\hbar} \int_{0}^{t} d t^{\prime}\left[\hat{H}_{T, I}(t),\left[\hat{H}_{T, I}\left(t^{\prime}\right), \hat{\rho}_{I}\left(t^{\prime}\right)\right]\right],
\end{aligned}
$$

which is still exact and allows a perturbative treatment in the tunneling Hamiltonian $\hat{H}_{T}$.

Prior to time $t=0$ the bath and the system do not interact, meaning that the total density matrix is factorized into a system and a leads component:

$$
\hat{\rho}_{I}(0)=\hat{\rho}_{S}(0) \hat{\rho}_{B}(0) .
$$

The density matrix of the leads, $\hat{\rho}_{B}$, can be described by the equilibrium thermodynamic expression shown in Eq. (22). Further we assume that the leads have so many degrees of freedom that they stay in thermal equilibrium up to a correction 
of order $\hat{H}_{T}$. It is convenient to trace out the degrees of freedom of the leads and define the reduced density matrix:

$$
\hat{\rho}_{\mathrm{red}, I}(t) \equiv \operatorname{Tr}_{\mathrm{B}} \hat{\rho}_{I}(t) .
$$

In the Schrödinger picture, the master equation for the reduced density matrix reads

$$
\begin{aligned}
\dot{\hat{\rho}}_{\text {red }}(t)= & \frac{i}{\hbar}\left[\hat{\rho}_{\text {red }}(t), \hat{H}_{S}\right]-\left(\frac{i}{\hbar}\right)^{2} \hat{U}_{0}(t) \int_{0}^{t} d t^{\prime} \\
& \times \operatorname{Tr}_{\mathrm{B}}\left(\left[\hat{H}_{T, I}(t),\left[\hat{H}_{T, I}\left(t^{\prime}\right), \hat{\rho}_{\mathrm{red}, I}\left(t^{\prime}\right) \hat{\rho}_{B}\right]\right]\right) \hat{U}_{0}^{\dagger}(t),
\end{aligned}
$$

where we neglect terms of order $\mathcal{O}\left(\hat{H}_{T}^{3}\right)$ and $\hat{U}_{0}(t)=e^{-(i / \hbar) \hat{H}_{S} t}$ is the time evolution operator of the unperturbed system.

\section{A. Superconducting leads}

The features of the superconducting leads are revealed when using the Bogoliubov transformation (6) to express the tunneling Hamiltonian. This yields additional terms compared to the normal conducting theory.

\section{Thermodynamic properties of the leads}

The description of electrons in terms of bogoliubons and Cooper pairs makes it necessary to discuss the thermodynamic properties of the superconducting leads. In this section we drop for simplicity the lead index $\eta$, and consider only one lead.

In order to calculate thermal expectation values we use the equilibrium density matrix of a superconductor:

$$
\hat{\rho}_{B}=\frac{e^{-\beta \hat{H}_{G}}}{Z_{G}},
$$

where $\hat{H}_{G}=\hat{H}_{B}-\mu \hat{N}, \beta$ is the inverse thermal energy, and $Z_{G}$ is the partition function in the grand canonical ensemble. We find that the thermal expectation value of a pair of Bogoliubov quasiparticles is equal to the Fermi function:

$$
\operatorname{Tr}_{\mathrm{B}}\left(\hat{\gamma}_{k \sigma}^{\dagger} \hat{\gamma}_{k \sigma} \hat{\rho}_{B}\right)=\frac{1}{e^{\beta E_{k}}+1}=f^{+}\left(E_{k}\right),
$$

where the trace is over the many-body states

$$
\left|\left\{n_{q \tau}\right\}, N\right\rangle=\prod_{q \tau}\left(\hat{\gamma}_{q \tau}^{\dagger}\right)^{n_{q \tau}}|0, N\rangle,
$$

with independent sums over the number of electrons $N$ in the Cooper pair condensate and the quasiparticle configuration $\left\{n_{q \tau}\right\}=\left\{n_{q_{1} \tau_{1}}, n_{q_{2} \tau_{2}}, \ldots\right\}$.

\section{Time evolution of the quasiparticles}

To proceed we have to specify the time evolution of the Bogoliubov and Cooper pair operators. We find

$$
\begin{gathered}
\hat{\gamma}_{\eta k \sigma, I}^{\dagger}(t)=e^{+(i / \hbar)\left(E_{k}+\mu_{\eta}\right) t} \hat{\gamma}_{\eta k \sigma}^{\dagger}, \\
\hat{S}_{\eta, I}^{\dagger}(t)=e^{+(i / \hbar) 2 \mu_{\eta} t} \hat{S}_{\eta}^{\dagger},
\end{gathered}
$$

in agreement with the results of Josephson and Bardeen. ${ }^{25,26}$ When calculating the time evolution it is important to remember that in the lead Hamiltonian the term $\mu_{\eta} \hat{N}_{\eta}$ is still written in terms of electron operators.
Before we proceed, we like to emphasize the importance of the Cooper pair contribution for finite bias voltages. As already pointed out by Governale et al. ${ }^{20}$ in this case $\mu_{\eta}$ cannot be set to zero and the time evolution of the Cooper pair operators, Eq. (26), plays an important role. Neglecting the Cooper pair contribution for finite bias voltages ${ }^{38}$ violates the number conservation in the tunneling processes and can lead to coherences which would vanish in the number conserving case.

\section{Difference to the normal conducting theory}

To compute Eq. (21) we rewrite the electron operators using the Bogoliubov transformation, Eq. (6), and insert the time evolution as in Eqs. (25) and (26). This yields four different traces to be calculated. We find

$$
\begin{aligned}
& \operatorname{Tr}_{\mathrm{B}}\left(\hat{c}_{\eta k \sigma, I}^{\dagger}(t) \hat{c}_{\eta^{\prime} k^{\prime} \sigma^{\prime}, I}\left(t^{\prime}\right) \hat{\rho}_{B}\right) \\
& =\delta_{\eta \eta^{\prime}} \delta_{k k^{\prime}} \delta_{\sigma \sigma^{\prime}}\left\{\left|u_{\eta k}\right|^{2} f^{+}\left(E_{\eta k}\right) e^{+(i / \hbar)\left(E_{\eta k}+\mu_{\eta}\right)\left(t-t^{\prime}\right)}\right. \\
& \left.+\left|v_{\eta k}\right|^{2} f^{-}\left(E_{\eta k}\right) e^{-(i / \hbar)\left(E_{\eta k}-\mu_{\eta}\right)\left(t-t^{\prime}\right)}\right\}, \\
& \operatorname{Tr}_{\mathrm{B}}\left(\hat{c}_{\eta k \sigma, I}(t) \hat{c}_{\eta^{\prime} k^{\prime} \sigma^{\prime}, I}^{\dagger}\left(t^{\prime}\right) \hat{\rho}_{B}\right) \\
& =\delta_{\eta \eta^{\prime}} \delta_{k k^{\prime}} \delta_{\sigma \sigma^{\prime}}\left\{\left|u_{\eta k}\right|^{2} f^{-}\left(E_{\eta k}\right) e^{-(i / \hbar)\left(E_{\eta k}+\mu_{\eta}\right)\left(t-t^{\prime}\right)}\right. \\
& \left.+\left|v_{\eta k}\right|^{2} f^{+}\left(E_{\eta k}\right) e^{+(i / \hbar)\left(E_{\eta k}-\mu_{\eta}\right)\left(t-t^{\prime}\right)}\right\}, \\
& \quad \operatorname{Tr}_{\mathrm{B}}\left(\hat{c}_{\eta k \sigma, I}^{\dagger}(t) \hat{c}_{\eta^{\prime} k^{\prime} \sigma^{\prime}, I}^{\dagger}\left(t^{\prime}\right) \hat{\rho}_{B}\right)=0, \\
& \quad \operatorname{Tr}_{\mathrm{B}}\left(\hat{c}_{\eta k \sigma, I}(t) \hat{c}_{\eta^{\prime} k^{\prime} \sigma^{\prime}, I}\left(t^{\prime}\right) \hat{\rho}_{B}\right)=0,
\end{aligned}
$$

where $f^{-}(E)=1-f^{+}(E)$. Note that the traces in Eqs. (29) and (30) are vanishing since the lead Hamiltonian, Eq. (16), conserves the particle number.

\section{B. General master equation for the reduced density matrix}

Collecting all the previous results and expressing Eq. (21) in the basis of the system eigenstates, $\{|n\rangle\}$, we obtain the Bloch-Redfield form of the general master equation (GME) for the reduced density matrix:

$$
\begin{aligned}
\dot{\rho}_{n n^{\prime}}= & -\frac{i}{\hbar}\left(E_{n}-E_{n^{\prime}}\right) \rho_{n n^{\prime}}(t) \\
& -\sum_{m m^{\prime}}\left(R_{n n^{\prime} m m^{\prime}}^{N \rightarrow N+1}+R_{n n^{\prime} m m^{\prime}}^{N \rightarrow N-1}\right) \rho_{m m^{\prime}}(t),
\end{aligned}
$$

where $n$ is a collective quantum number of the many-body states of the quantum dot system and $\rho_{n n^{\prime}} \equiv\left\langle n\left|\hat{\rho}_{\text {red }}\right| n^{\prime}\right\rangle$. Here, the Redfield tensors are defined as

$$
\begin{aligned}
R_{n n^{\prime} m m^{\prime}}^{N \rightarrow} & =\sum_{\eta}\left\{\delta_{m^{\prime} n^{\prime}} \sum_{l}\left(\Gamma_{n l l m}^{+}\right)_{\eta}^{N \rightarrow N \pm 1}+\delta_{m n} \sum_{l}\left(\Gamma_{m^{\prime} l l n^{\prime}}^{-}\right)_{\eta}^{N \rightarrow N \pm 1}\right. \\
& \left.-\left(\Gamma_{m^{\prime} n^{\prime} n m}^{+}\right)_{\eta}^{N \rightarrow N \pm 1}-\left(\Gamma_{m^{\prime} n^{\prime} n m}^{-}\right)_{\eta}^{N \rightarrow N \pm 1}\right\}
\end{aligned}
$$

The rates $\Gamma$ in Eq. (32) originate from terms containing traces of the type of Eqs. (27) and (28). Further, we distinguish between rates describing the increase and rates describing the decrease of the particle number on the system, emphasized with the superscript $N \rightarrow N \pm 1$. Their detailed form is presented 
in Appendix B. The rates with the superscripts \pm are connected by complex conjugation and reversing of the indices:

$$
\left(\Gamma_{n m m^{\prime} n^{\prime}}^{-}\right)_{\eta}^{N \rightarrow N \pm 1}=\left(\left(\Gamma_{n^{\prime} m^{\prime} m n}^{+}\right)_{\eta}^{N \rightarrow N \pm 1}\right)^{*} .
$$

\section{Current}

Having derived the GME for the reduced density matrix in Eq. (31), we can use it to calculate measurable quantities such as the current and the differential conductance. In this section we present an expression for the current derived from the second order GME of Eq. (31). To do this we introduce a current operator whose statistical average gives the total current:

$$
I_{\eta}=\operatorname{Tr}\left(\hat{I}_{\eta} \hat{\rho}_{\text {tot }}\right)
$$

In general, the current operator of lead $\eta$ is defined as the variation of the total particle number in lead $\eta$ with time:

$$
\hat{I}_{\eta, I}(t)=-e \frac{d}{d t} \hat{N}_{\eta, I}(t)=\frac{+i e}{\hbar}\left[\hat{N}_{\eta, I}(t), \hat{H}_{T, I}(t)\right] .
$$

Calculating the commutator of Eq. (35), we see that the current operator has the same operatorial structure as the tunneling Hamiltonian:

$$
\begin{aligned}
\hat{I}_{\eta, I}(t)= & \frac{+i e}{\hbar} \sum_{k \alpha}\left(t_{\eta \alpha \sigma} \hat{c}_{\eta k \sigma, I}^{\dagger}(t) \hat{d}_{\alpha \sigma, I}(t)\right. \\
& \left.-t_{\eta \alpha \sigma}^{*} \hat{d}_{\alpha \sigma, I}^{\dagger}(t) \hat{c}_{\eta k \sigma, I}(t)\right),
\end{aligned}
$$

differing only in the prefactor and summation. Hence, by applying the same perturbation theory as before, we obtain for the current in lead $\eta$

$$
I_{\eta}(t)=e \sum_{n m l}\left(\left(\Gamma_{n l l m}^{N \rightarrow N+1}\right)_{\eta}-\left(\Gamma_{n l l m}^{N \rightarrow N-1}\right)_{\eta}\right) \rho_{m n}^{N}(t) .
$$

In Eq. (37) we introduced the abbreviations

$$
\begin{aligned}
\left(\Gamma_{n m m^{\prime} n^{\prime}}^{N \rightarrow N \pm 1}\right)_{\eta} & =\left(\Gamma_{n m m^{\prime} n^{\prime}}^{+}\right)_{\eta}^{N \rightarrow N \pm 1}+\left(\Gamma_{n m m^{\prime} n^{\prime}}^{-}\right)_{\eta}^{N \rightarrow N \pm 1} \\
& =2 \operatorname{Re}\left(\left(\Gamma_{n m m^{\prime} n^{\prime}}^{+}\right)_{\eta}^{N \rightarrow N+1}\right),
\end{aligned}
$$

exploiting Eq. (33). This gives us rates which are real and read

$$
\begin{aligned}
\left(\Gamma_{n m m^{\prime} n^{\prime}}^{N \rightarrow 1}\right)_{\eta}= & \operatorname{Re}\left(\tilde{\Gamma}_{n m m^{\prime} n^{\prime}}^{\eta} D\left(E_{m^{\prime} n^{\prime}}-\mu_{\eta}+i \gamma\right)\right. \\
& \left.\times f^{+}\left(E_{m^{\prime} n^{\prime}}-\mu_{\eta}+i \gamma\right)\right), \\
\left(\Gamma_{n m m^{\prime} n^{\prime}}^{N \rightarrow N-1}\right)_{\eta}= & \operatorname{Re}\left(\tilde{\Gamma}_{m^{\prime} n^{\prime} n m}^{\eta} D\left(E_{n^{\prime} m^{\prime}}-\mu_{\eta}+i \gamma\right)\right. \\
& \left.\times f^{-}\left(E_{n^{\prime} m^{\prime}}-\mu_{\eta}+i \gamma\right)\right),
\end{aligned}
$$

where

$$
\tilde{\Gamma}_{n m m^{\prime} n^{\prime}}^{\eta}=\frac{2 \pi}{\hbar} \sum_{\sigma \alpha \alpha^{\prime}} t_{\eta \alpha \sigma} t_{\eta \alpha^{\prime} \sigma}^{*}\left\langle n\left|\hat{d}_{\alpha \sigma}\right| m\right\rangle\left\langle m^{\prime}\left|\hat{d}_{\alpha^{\prime} \sigma}^{\dagger}\right| n^{\prime}\right\rangle .
$$

In Eqs. (39) and (40) $E_{n^{\prime} m^{\prime}}=E_{n}^{\prime}-E_{m}^{\prime}$ denote differences between system eigenenergies and

$$
D(E)=\rho_{N} \operatorname{Re}\left(\frac{|E|}{\sqrt{E^{2}-|\Delta|^{2}}}\right)
$$

is the BCS density of states, with $\rho_{N}=\frac{V m k_{F}}{2 \pi^{2} \hbar^{2}}$ labeling the density of states for normal leads which is assumed to be constant around the Fermi level; $V$ denotes the volume of the lead and $m$ is the electron mass. In order to renormalize the divergence of the density of states we introduced a finite lifetime $\hbar / \gamma$ of the quasiparticle states in the superconducting leads, leading to a Lorentzian broadening of the resonance condition (see Appendix B 2). This assumption is also in agreement with the results of Levy Yeyati et al., ${ }^{17}$ where they showed that the broadening of the BCS-like features in the current is due to the coupling to the leads. Equation (37) is a general result and can be applied to any transport setup where an arbitrary system with discrete levels is weakly coupled to superconducting or normal conducting leads. The normal conducting case is obtained by setting $\left|\Delta_{\eta}\right|=0$ and $\gamma=0$.

The theory is valid in the so-called weak-coupling limit, which is defined by the following relations between fundamental energy scales of the system: $\Gamma \ll|\Delta| \ll U$ and $\Gamma \ll k_{B} T$, where $\Gamma$ is the level broadening due to hybridization with the leads, $U$ is the charging energy, and $|\Delta|$ is the superconducting gap. As proven, for example in Ref. 17, the inclusion of higher order terms only produces in this regime an effective broadening of the quasiparticle density of states without invalidating the sequential tunneling description.

In this paper we are only interested in the stationary limit. Hence, we replace the density matrix in Eq. (37) by its stationary solution which is determined from Eq. (31) by imposing $\dot{\rho}_{n n^{\prime}}^{N}=0$.

\section{TRANSPORT THROUGH MULTIPLE QUANTUM DOT DEVICES}

In the preceding sections we developed a perturbative microscopic theory for the stationary current of quantum dot devices coupled to superconducting leads. In the following, we show the predictions of the theory for two models, the SD and the DD. In the transport setup the bias and gate voltages influence the energy configuration of the leads and the system, respectively. Specifically, the bias voltage is modifying the electrochemical potential of the leads, which we choose to have a symmetric voltage drop. Therefore we define the chemical potentials of the left and right leads, respectively:

$$
\mu_{L / R}=\mu_{0} \pm e \frac{V_{b}}{2},
$$

where $\mu_{0}$ is the equilibrium chemical potential. The gate voltages are modifying the on-site energies of the system: We replace $\epsilon_{d} \rightarrow \epsilon_{d}+e V_{g}$ in the $\mathrm{SD}$ and $\epsilon_{\alpha} \rightarrow \epsilon_{\alpha}+e V_{g}^{\alpha}$ in the DD Hamiltonian. Here $e=-|e|$ is the electron charge.

In the following we neglect coherences in the GME, considering only diagonal contributions of the reduced density matrix $\rho_{n n}$ by setting $n=n^{\prime}$ in Eq. (31). Hence, it suffices to use only two indices for the transition rates.

Neglecting the coherences is a nontrivial step in the derivation of the master equation for the system. Within the secular approximation (see Ref. 37), justified in the weakcoupling limit, only coherences between degenerate states can play a role. We can now distinguish three types of degeneracies in the many-body spectrum of a quantum dot molecule: spin degeneracy, orbital degeneracy, and degeneracy between states with different particle numbers. Spin degeneracies can be neglected in the presence of unpolarized or collinearly polarized leads. ${ }^{30,39}$ Orbital degeneracies are system dependent and they 
are not present in the single and double quantum dot systems discussed in this paper. A detailed discussion of their effects can be found, for example, in Refs. 39 and 40. A detailed analysis of Eq. (31) shows that only "anomalous" terms originating from Eqs. (29) and (30) could couple populations $\left(\rho_{N, N}\right)$ with coherences $\left(\rho_{N-1, N+1}\right)$. Since these terms are exactly vanishing in the number conserving description of the superconducting leads, coherences decouple from populations and vanish in the stationary limit due to the damping introduced by the "R" components.

In current-voltage spectroscopy it is convenient to illustrate the conditions under which current is allowed to flow as lines in the stability diagrams. These so-called transition lines are fixed by the energetic part of the transition rates at the source $\eta=S$ and the drain $\eta=D$ contact:

$$
\begin{aligned}
& \left(\Gamma_{m n}^{N \rightarrow N+1}\right)_{\eta} \propto f^{+}\left(\Delta E-\mu_{\eta}\right) D\left(\Delta E-\mu_{\eta}\right), \\
& \left(\Gamma_{n m}^{N+1 \rightarrow N}\right)_{\eta} \propto f^{-}\left(\Delta E-\mu_{\eta}\right) D\left(\Delta E-\mu_{\eta}\right),
\end{aligned}
$$

neglecting the lifetime broadening $\gamma$ for simplicity, and with $\Delta E=E_{m}^{N+1}-E_{n}^{N}$ the energy difference of the two transport levels. Figure 2 illustrates this product for two different temperatures: For high enough temperatures quasiparticles can be excited thermally across the gap giving a small peak in the transition rates. ${ }^{16}$ The peak positions define transition lines when plotted in a $V_{g}-V_{b}$ diagram. Notice that while the most pronounced peak survives also at zero temperature and defines a transport threshold, the second peak vanishes at low temperatures and essentially only processes at and close to the peak are relevant. For an $N \rightarrow N+1$ transition we denote transitions associated to the more pronounced peak as $\mathrm{S}+$ and $\mathrm{D}+$ when happening at the source or at the drain contact, respectively. Transitions involving thermally excited quasiparticles are called $\mathrm{St}+$ and $\mathrm{Dt}+$. In complete analogy, we classify transitions from $N+1 \rightarrow N$ : We denote by $\mathrm{S}-$ and $\mathrm{D}-$ the more pronounced transitions at the source and at the drain, and by $\mathrm{St}-$ and $\mathrm{Dt}-$ their thermal counterparts.
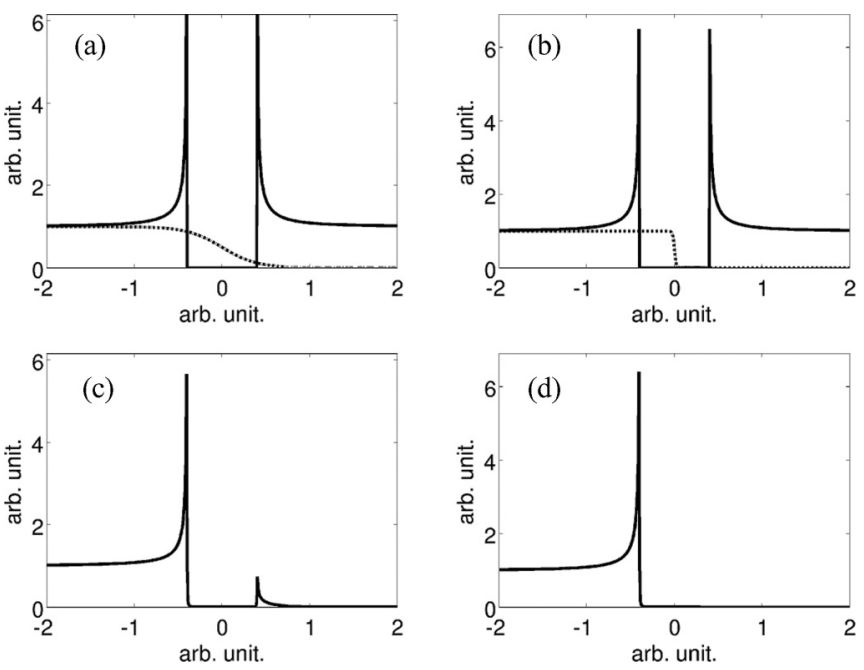

FIG. 2. (a) and (b) Density of states (continuous line) and Fermi function (dotted line) at $k_{B} T=0.2 \mathrm{meV}$ and $k_{B} T=0.01 \mathrm{meV}$, respectively. (c) and (d) Product of the density of states and the Fermi function for the temperatures used in (a) and (b), respectively.

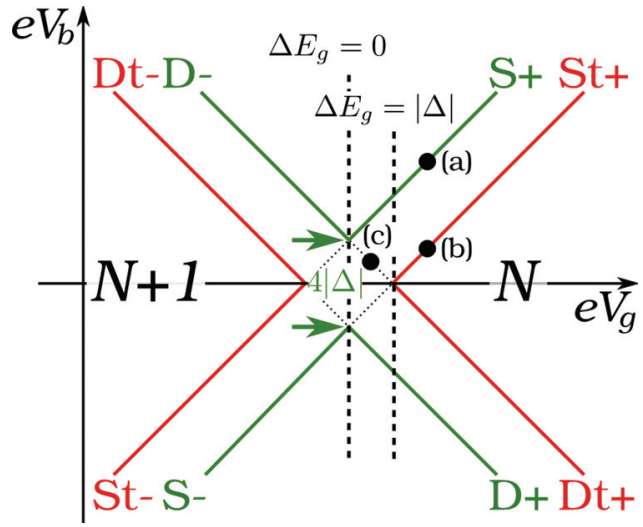

FIG. 3. (Color online) Illustration of the transition lines appearing in the presence of superconducting leads. The green lines mark transitions at the source and the drain contacts, described by the inequalities of Eqs. (46), (47), (50), and (51). The red lines mark transitions involving thermally excited quasiparticles, given by Eqs. (48), (49), (52), and (53). The $E_{g}-N$ diagrams for the points (a)-(c) are sketched in Fig. 5.

In total we find eight different transition lines, as depicted in Fig. 3. In the following we derive transport conditions and provide equations for the transport lines. For convenience we introduce $\Delta E_{g}=\Delta E-\mu_{0}$.

We start with the analysis of the $N \rightarrow N+1$ transitions, which are described by the rates in Eq. (44). From the arguments we find that the rates do not vanish if

$$
\begin{array}{cc}
\Delta E_{g} \leqslant-|\Delta|+\frac{e V_{b}}{2}, & \text { Source } \mathrm{S}+, \\
\Delta E_{g} \leqslant-|\Delta|-\frac{e V_{b}}{2}, & \text { Drain D+. }
\end{array}
$$

Another contribution comes from the thermally excited quasiparticle states, namely, if the argument of the Fermi function $f^{+}\left(\Delta E-\mu_{\eta}\right)$ and of the density of states $D\left(\Delta E-\mu_{\eta}\right)$ is equal to $|\Delta|$. At this point the transition rates are peaked and contribute to the current:

$$
\begin{array}{ll}
\Delta E_{g}=|\Delta|+\frac{e V_{b}}{2}, & \text { Source thermal St }+, \\
\Delta E_{g}=|\Delta|-\frac{e V_{b}}{2}, & \text { Drain thermal Dt+. }
\end{array}
$$

Since the thermally excited quasiparticles produce a peak rather than a step in the current-voltage characteristic, the corresponding transport condition is formulated with an equality.

Transitions from $N+1 \rightarrow N$ are described by the rate of Eq. (45), leading in complete analogy to the previous case to the following transport conditions:

$$
\begin{gathered}
-\Delta E_{g} \leqslant-|\Delta|-\frac{e V_{b}}{2}, \quad \text { Source } \mathrm{S}-, \\
-\Delta E_{g} \leqslant-|\Delta|+\frac{e V_{b}}{2}, \quad \text { Drain D-, } \\
-\Delta E_{g}=|\Delta|-\frac{e V_{b}}{2}, \quad \text { Source thermal St- } \\
-\Delta E_{g}=|\Delta|+\frac{e V_{b}}{2}, \quad \text { Drain thermal Dt-. }
\end{gathered}
$$



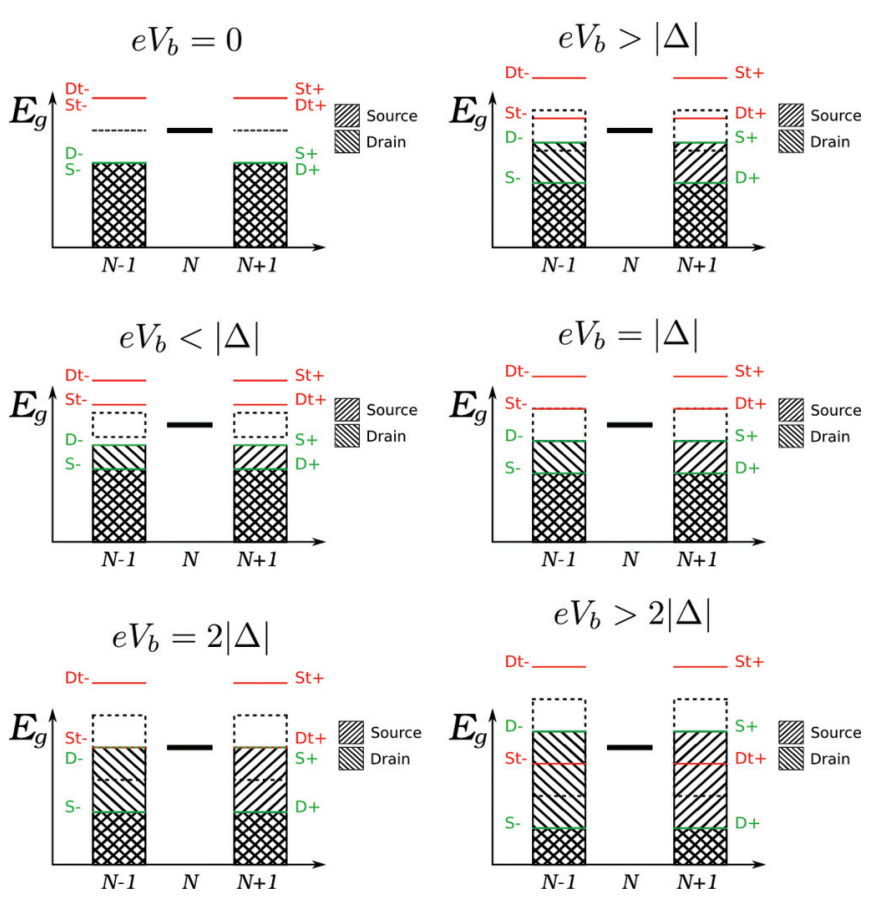

FIG. 4. (Color online) Visualization of the transport conditions of Eqs. (46)-(53). We plotted the threshold of the transport inequalities as green lines $(\mathrm{S} \pm, \mathrm{D} \pm)$; for the equalities coming from transitions involving thermally excited quasiparticles we used red lines ( $\mathrm{St} \pm$, Dt \pm ). Choosing the reference level in the $N$-particle subspace, we found a scheme where transitions are energetically allowed to levels which lie in the shaded region below the green lines and to levels which align with the red lines. Dashed boxes mark the bias window $e V_{b}$.

Visualization of the transport conditions. To visualize the transport conditions of Eqs. (46)-(53) we extend the scheme of Donarini et al. of Ref. 39 to superconducting leads. The scheme is depicted in Fig. 4 and illustrates for which relative position of the systems eigenenergies $E_{g}^{N}=E_{m}^{N}-\mu_{0} N$ transitions are energetically allowed. The bias window is marked with a dashed box. The green lines mark the borders of the inequalities, and the red lines the sharp equalities for the thermal transitions, meaning that transitions can occur to states lying below the green lines (shaded region), and to states which coincide with the red lines. In order to see a transition between two levels in the stability diagram a source and a drain transition must be allowed between the two levels (depicted as arrows in the $E_{g}-N$ diagrams of Fig. 5). We note that for a full analysis of the transport properties also the geometrical part of the rates must be taken into account and transport occurs only if $\tilde{\Gamma} \neq 0$.

\section{A. Single level quantum dot model}

The simplest example of a quantum dot system is the single level quantum dot presented in Eq. (2). Since only one level is involved, we can do most calculations analytically and understand the basic mechanism resulting from the superconducting leads. In Fig. 6 the stationary current is shown as a function of bias and gate voltage for superconducting leads at $k_{B} T=0.5|\Delta|$. We observe the expected gap 5 between the Coulomb diamonds which is equal to $4|\Delta| / e$. The gap can be explained using Fig. 3 and the corresponding Eqs. (46)-(53). (a)

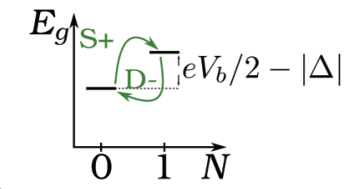

(c)

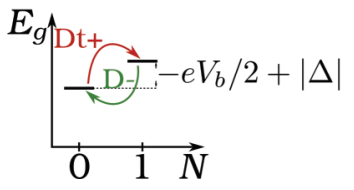

(b)

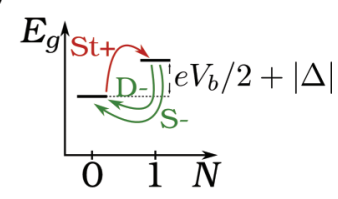

FIG. 5. (Color online) (a)-(d) $E_{g}-N$ diagrams for a single level quantum dot with $\Delta E_{g}>|\Delta|$ and at bias voltages as sketched in Fig. 3. For the simulations of Fig. $6 \Delta E_{g}>\Delta$ corresponds to a gate voltage $e V_{g}<-2.6 \mathrm{meV}$. In (a) we cut the $\mathrm{S}+$ line: The particle number on the system is increased by a tunneling event at the source contact and decreased at the drain. (b) Cut with the thermal line $\mathrm{St}+$ : The particle number of the system is increased by a tunneling event involving a thermally excited quasiparticle at the source contact and decreased by tunneling into empty states in the source and the drain contact, respectively. (c) $E_{g}-N$ diagrams for a single level with $0<\Delta E_{g}<|\Delta|$. The two levels are only connected by two drain transitions, meaning that in this configuration the system is in thermal equilibrium with the drain contact.

One dashed line marks the gate voltage where $\Delta E_{g}=0$. Along this line the conditions under which current is allowed to flow read $e V_{b} / 2>|\Delta|$ for the $\mathrm{S}+, \mathrm{D}-$ lines, and $e V_{b} / 2<-|\Delta|$ for the $\mathrm{S}-, \mathrm{D}+$ lines, opening a bias window of $4|\Delta| / e$ where current is blocked for low temperatures $k_{B} T \ll|\Delta|$. For higher temperatures of $k_{B} T \approx 0.5|\Delta|$ we observe small peaks in the Coulomb blockade region (green area) which are due to thermally excited quasiparticles; they correspond to the red lines in Fig. 3. In Fig. 5 we show the energy particle number diagrams in the points (a)-(d), which lie on a vertical cut through Fig. 3 at $\Delta E_{g}>|\Delta|$ which corresponds to a gate voltage $e V_{g}>2.6 \mathrm{meV}$ in Fig. 6. In Fig. 5(a) we depicted the $E_{g}-N$ diagram for a cut with the $\mathrm{S}+$ resonance line, where the particle number on the system is increased by a tunneling event at the source and decreased at the drain contact. For bias voltages smaller than the one at resonance (corresponding to larger $e V_{b}$ as $e$ is the negative charge of an electron) the $\mathrm{S}+, \mathrm{D}-$ transitions remain open and current can flow. In Fig. 5(b) the $E_{g}-N$ diagram at the resonance line $\mathrm{St}+$ is
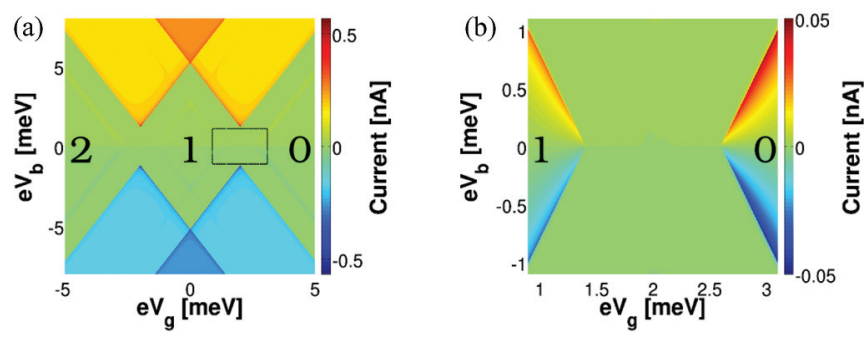

FIG. 6. (Color online) (a) Current-voltage characteristics of a SD coupled to superconducting leads. Parameters are $k_{B} T=0.3 \mathrm{meV}$ and $|\Delta|=0.6 \mathrm{meV}, \quad U=4 \mathrm{meV}, \epsilon_{d}=-2 \mathrm{meV}$, and $e \Gamma=$ $0.001 \mathrm{meV}$. (b) Subgap features coming from thermally excited quasiparticles of the 0-1-particle transition, highlighted as a dashed box in (a). 
shown. In this case the bias voltage is not large enough to allow the transitions $\mathrm{S}+$ of Eq. (46). For low temperatures no quasiparticle is thermally excited meaning that only transitions from $1 \rightarrow 0$ are energetically allowed (green arrows). For high enough temperatures, however, the particle number of the system can be increased by tunneling events involving thermally excited quasiparticles opening the $\mathrm{St}+$ transition. By changing the sign of the bias voltage the role of the source and the drain is inverted, explaining the transition lines Dt+ and $\mathrm{D}+$ [Figs. 5(c) and 5(d)].

Another interesting constellation of the energy level occurs in the region of $0<\Delta E_{g}<|\Delta|$ [Fig. 5(e)], where in the current-voltage characteristics the thermal lines are vanishing. Transitions can only occur at the drain contact, as the bias is not large enough to allow transitions at the source. Hence, the system is in thermal equilibrium with the drain contact and the occupation probabilities are related by the Boltzmann distribution:

$$
\frac{\rho_{0}}{\rho_{1}}=e^{\beta\left(\Delta E_{g}+e V_{b} / 2\right)},
$$

in the limit of $\gamma \rightarrow 0$.

\section{B. The double quantum dot}

We have seen that the theory can reproduce well-known results for the SD and we understood the properties of the thermal transitions in $E_{g}-N$ diagrams with only one nondegenerated level per particle number. In the following we investigate a more advanced system, the double quantum dot, where the many-body spectrum gives rise to more than one nondegenerated level per particle number, so-called excited system states. For normal conducting leads the excitations cannot be seen for low bias voltages, since transitions to the ground state are always possible, blocking transport through the excitations. In the last section we have seen that for superconducting leads the energy difference must be at least $\left|\Delta E_{g}\right| \geqslant e V_{b} / 2-|\Delta|$ to have nonthermal source and drain transitions. Hence, we find situations where the transitions to the ground state are energetically not allowed and transport occurs through excited system states.

We start with equally gated dots with the same on-site energies and on-site Coulomb interactions, where it is possible to diagonalize the Hamiltonian analytically. ${ }^{29,30}$ In the second part, the case of independently coupled dots is discussed, where the detuning of the two gate voltages influences the level spacing of the energy spectrum. Thus, excited states can be observed only in detuning ranges where the difference between the energy level of the excited state and its ground state is less than $2|\Delta|$.

\section{Equally gated dots}

For equally gated dots the on-site energies of the two sites are modulated with the same gate voltage. Hence, it is convenient to plot the current as a function of the bias and the gate voltage as for the SD. Figure 7 shows the current of an equally gated DD in serial configuration. As for the SD we observe Coulomb blockade and the gap of $4|\Delta| / e$ between the tips of the diamonds. Transport carried by thermally excited quasiparticles is of particular interest, as it allows one to observe transitions through excited system
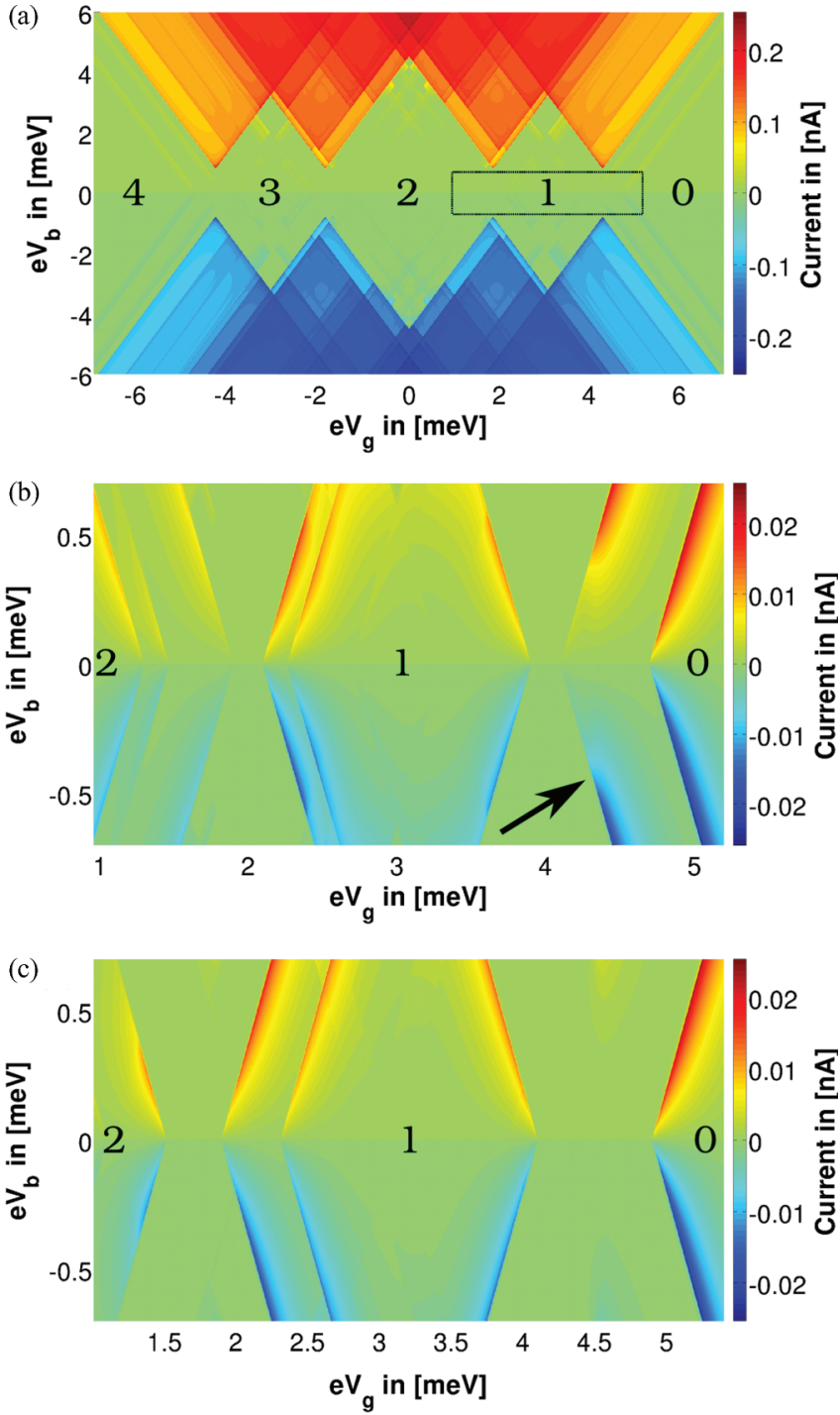

FIG. 7. (Color online) (a) Current-voltage characteristics of an equally gated DD in serial configuration at $k_{B} T=0.2 \mathrm{meV}$, $|\Delta|=0.4 \mathrm{meV}, U=4 \mathrm{meV}, V=2 \mathrm{meV}, \quad b=-0.3 \mathrm{meV}$, and $e \Gamma=0.001 \mathrm{meV}$. (b) $I-V$ characteristics in the subgap region corresponding to the dashed box in (a). The distance between the 1-particle excited state and its ground state is equal to the coupling strength $2|b|$ of the two dots. Moreover, $2|b|<2|\Delta|$. The black arrow marks the transition line coming from transport through the 1-particle excited state. (c) $I-V$ characteristics in the subgap region, where we increased the coupling between the two dots $(b=-0.5 \mathrm{meV})$, leading to a level spacing which is larger than $2|\Delta|$, hence transport through the excited system state is not allowed and the line disappears.

states for low bias voltages, which are often diminished by the ground state transitions in the normal conducting case. In order to show some interesting phenomena resulting from the more complex spectrum, we concentrate on the 0 to 1-particle transition where three levels are involved. In the 1-particle spectrum, the difference between the ground state and the excited state is equal to $2|b|$, where $b<0$ is the tunneling strength between the two dots. Meaning that by tuning the coupling between the two dots it is possible to influence the level spacing. Figure 8 shows a sketch of the 


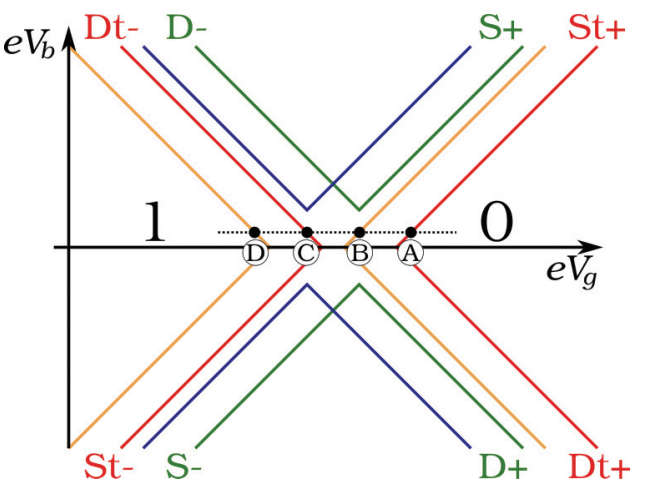

FIG. 8. (Color online) Sketch of the transition lines for the 0-1particle transition of an equally gated DD. It shows two copies of Fig. 3 where the labeling of the blue (orange lines) is the same as for the green (red) lines. The blue (orange) lines mark the transition lines corresponding to the 0 -particle ground state to 1-particle first excited state transition.

transition lines expected for the $0-1$ transition for $|b|<|\Delta|$, where the red (green) lines show the ground state to ground state transitions, and the blue (orange) lines the ground state to first excited state transitions. For a better understanding of the transport properties we cut the transition lines horizontally for a small bias voltage $e V_{b} / 2<|\Delta|$ in the Coulomb blockade region [points $\mathrm{A}-\mathrm{D}$ ]; the corresponding $E_{g}-N$ diagrams are depicted in Fig. 9. In point $\mathrm{A}$ the difference between the ground states is equal to $\Delta E_{g}=e V_{b} / 2+|\Delta|$ opening the thermal transition $\mathrm{St}+$ and current can flow. Following the dashed line to point $\mathrm{B}$, the 1-particle states are shifted down in energy until the $\mathrm{St}+$ transition is allowed between the 0-particle
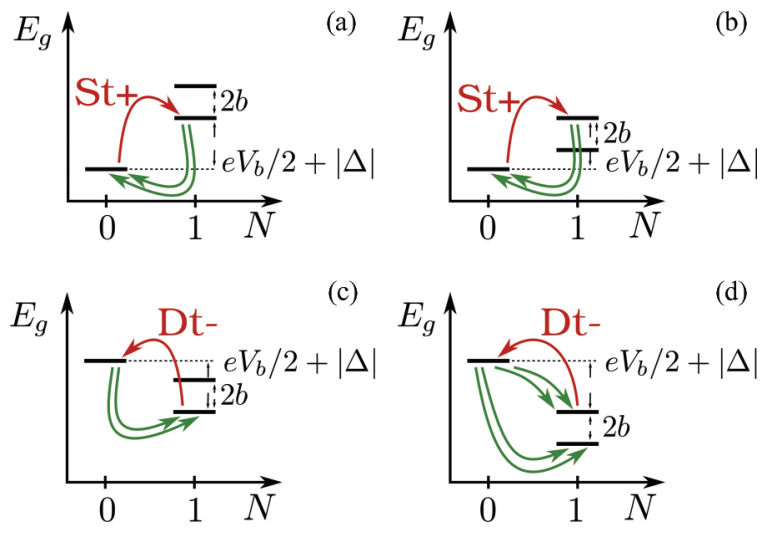

FIG. 9. (Color online) $E_{g}-N$ diagram corresponding to the points of Fig. 8 where the dashed line cuts the transition lines for the case of an equally gated DD. In this case the distance between the 1-particle ground state to the 1-particle first excited state is equal to $2 b<2|\Delta|$, where $b$ is the tunneling strength between the two quantum dots. (a) Point on the thermal line $\mathrm{St}+$ of the ground state to ground state transition. (b) Point on the thermal line St+ of the ground state to first excited state transition. (c) Point on the Dt- line of the ground state to ground state transition. (d) Point on the Dt- line of the ground state to first excited state transition; this line cannot be seen in the current-voltage characteristics, as the ground state to ground state transitions are open. Hence, in the long-time behavior the system will occupy the 1-particle ground state blocking the current through the excited state.

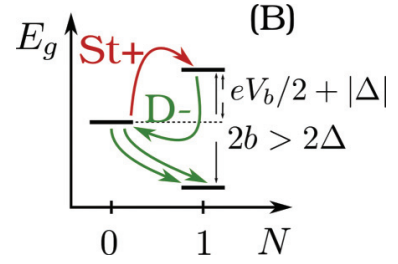

FIG. 10. (Color online) $E_{g}-N$ diagram of point B in Fig. 8, for a level spacing of the 1-particle energies larger than $2 b>2 \Delta$. In contrast to Fig. 9 the transition between the 0-particle ground state and the 1-particle excited state is open, blocking the current.

ground state and the 1-particle excited state. Since $|b|<\Delta$, the 1-particle ground state is energetically not accessible and current can flow through the excited state. We like to emphasize that the blocking of the ground state transition is only valid as long as the distance between the two 1-particle levels is smaller than $2|\Delta|$. For larger distances the ground state is energetically accessible, blocking the current through the excited state (cf. Fig. 10). In point $\mathrm{C} e V_{g}$ is further decreased, the $\mathrm{Dt}-$ transition between the ground states is opening, and current can flow. Point D shows the typical energy configuration in which current through the excited state is blocked, even though the transition through the excited state is energetically allowed. The reason for that is the 1-particle ground state which can be populated, but transitions describing its depopulation are energetically not allowed, leading to a blocking of the current in the stationary limit.

To demonstrate the important role of the level spacing we show the current-voltage characteristics of an equally gated DD in the subgap region in Figs. 7(b) and 7(c). In (b) the spacing of the 1-particle energy levels $|2 b|<2|\Delta|$, hence, the excited state can be observed in the current (arrow in Fig. 7). In (c) we increase the tunneling strength between the two dots $2|b|>2|\Delta|$ and the excited state line is vanishing, as explained in Fig. 10. As in the case for $2|b|<2|\Delta|$ the excited level is in resonance with the $\mathrm{St}+$ transition, however, due to the larger level spacing, the ground state transition opens and current is blocked.

\section{Independently gated dots}

In the last paragraph we considered a DD with both dots coupled to the same gate electrode. In most experiments, however, it is more convenient to couple the dots independently, which leads to a "honeycomb"-shaped current-voltage characteristic. ${ }^{41}$ For symmetric on-site energies and Coulomb repulsion it is possible to diagonalize the DD Hamiltonian of Eq. (3) analytically. Gating the dots independently destroys this symmetry, an analytic diagonalization is not possible, and one has to use numerical methods. We plot the current as a function of the detuning $\Delta_{g}=V_{g}^{1}-V_{g}^{2}$, and the average of the two gate voltages $\Sigma_{g}=\left(V_{g}^{1}+V_{g}^{2}\right) / 2$.

The current-voltage characteristic for serial and parallel configurations is depicted for the normal conducting case in Figs. 11(a) and 11(b) and for the superconducting case in Figs. 11(c) and 11(d). Comparing both configurations, we observe for the serial one a decrease in the current for high detuning $\Delta_{g}$, while in the parallel configuration current can be observed over the entire voltage range. This difference is a 

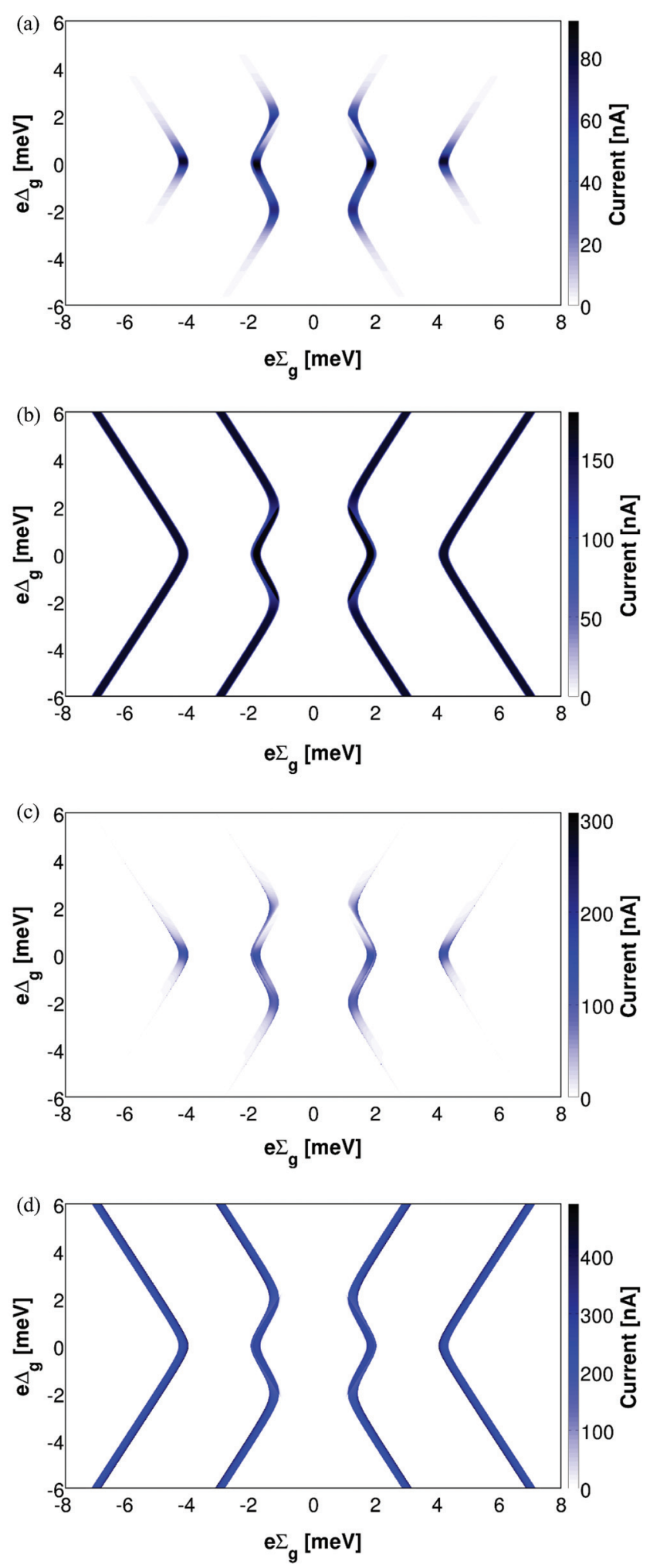

FIG. 11. (Color online) (a), (b) Current-voltage characteristics of a DD coupled to normal conducting leads in serial (a) and in parallel (b) configurations. We fixed the bias voltage to $e V_{b}=0.3 \mathrm{meV}$. (c), (d) Current-voltage characteristics of a DD coupled to superconducting leads in serial (c) and in parallel (d) configurations. We fixed the bias voltage to $e V_{b}=0.3 \mathrm{meV}+2|\Delta|$ in order to obtain the same conditions as for the normal conducting case in (a) and (b). Parameters are $T=0.01 \mathrm{meV},|\Delta|=0.4 \mathrm{meV}, e \Gamma=0.001 \mathrm{meV}$, $b=-0.2 \mathrm{meV}, U=4 \mathrm{meV}$, and $V=2 \mathrm{meV}$. (a)

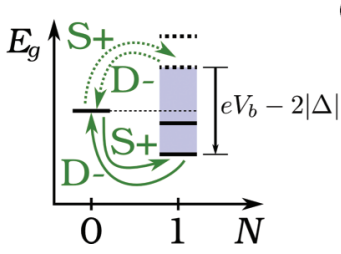

(b)

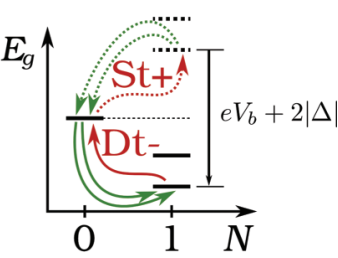

FIG. 12. (Color online) (a) $E_{g}-N$ diagram of the 0-1-particle transition for $e V_{b} / 2>|\Delta|$. In the 1-particle spectrum we plotted two situations which mark the borders of the current step. The dashed levels mark the left border (for small $\Sigma_{g}$ ) where the 1-particle levels lie above the 0-particle energy level. If the distance $\Delta E_{g} \leqslant e V_{b} / 2-|\Delta|$, current can flow through $\mathrm{S}+$ and $\mathrm{D}-$ transitions. By lowering $e \Sigma_{g}$ the 1-particle energy levels move down in the $E_{g}-N$ diagram, while the transitions remain open. The solid lines mark the right border of the current steps, as for levels lying below the solid line the D- transition is closed and current is blocked. Thus, the width of the current steps in the current-voltage characteristics is $e \Delta \Sigma_{g}=$ $e V_{b}-2|\Delta|$. (b) $E_{g}-N$ diagram of the 0-1-particle transition involving thermal transitions. For the same arguments as in (a), the distance between two thermal lines in the current-voltage characteristics is equal to $e \Delta \Sigma_{g}=e V_{b}+2|\Delta|$.

consequence of the geometry of the setup as the DD system remains unchanged. An increase of the detuning leads to a localization of the systems ground state at site 1 and transitions through site 2 are blocked. Since in serial configuration the right lead is only coupled to site 2 , the localization of the wave function at site 1 leads to a decrease in the current. In parallel configuration, however, both sites are coupled to both leads and the ground state transition is always open.

The left and right borders of the current steps are given by the source and drain lines, respectively. They follow, in complete analogy to the simplest case, from energy conservation. In Fig. 12(a) we show the $E_{g}-N$ diagram for the 0 - to 1-particle transition illustrating two limits: The ground states are (i) in resonance with the $\mathrm{S}+$ transition (dashed line) and (ii) in resonance with the $\mathrm{D}-$ transition (solid line), describing the left and right borders of the current step in Figs. 11(c) and 11(d). Starting at the $\mathrm{S}+$ resonance, the energy levels of the 1-particle spectrum are moving down in energy by increasing the average gate voltage $\Sigma_{g}$. Both transitions (S+ and $\mathrm{D}-$ ) remain open as long as the ground state lies in the blue (shaded) region. If the ground state lies below the solid line, the D- transition is closed and current is blocked. Hence, the width of the current steps in the current-voltage characteristics in Figs. 11(c) and 11(d) is equal to the size of the blue (shaded) region in Fig. 12(a), namely, $e \Delta \Sigma_{g}=e V_{b}-2|\Delta|$. The same arguments hold for the distance of two corresponding thermal transitions; as illustrated in Fig. 12(b) the distance of two thermal lines is equal to $e \Delta \Sigma_{g}=e V_{b}+2|\Delta|$.

As we can see in Fig. 11 there exists a one-to-one correspondence of the transport conditions of the normal conducting to the superconducting case, which leads to the same shape of the current-voltage characteristics if $k_{B} T \ll|\Delta|$. Increasing the bias voltage by $2|\Delta|$ compared to the normal conducting case $e V_{b}^{\mathrm{SC}}=e V_{b}^{\mathrm{NC}}+2|\Delta|$ leads to the same transport conditions. Although the shape of the current steps in Figs. 11(a) and 11(b) and Figs. 11(c) and 11(d) look the same, they differ at the edges of the current steps, as in the superconducting case the sharp 


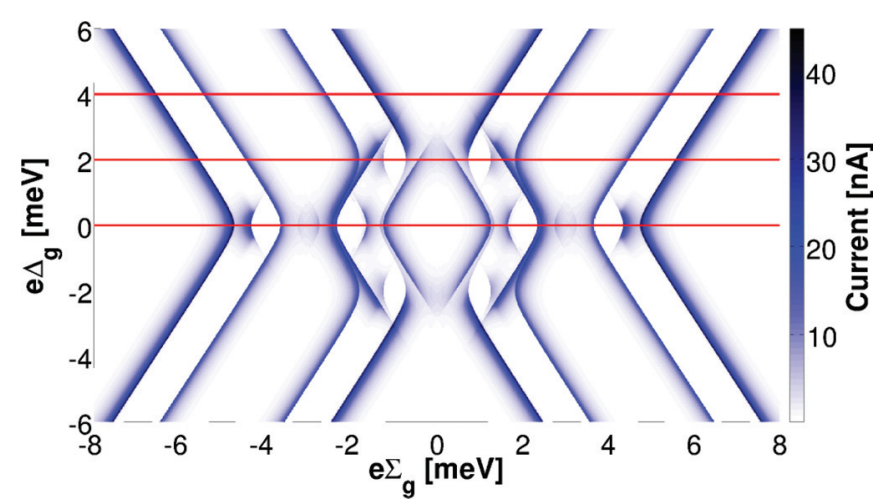

FIG. 13. (Color online) Current-voltage characteristics of a DD in parallel configuration for bias $V_{b}<2|\Delta| / e$. Since the bias voltage is not high enough current can flow only due to thermally excited quasiparticles. The red lines correspond to Fig. 15 where the energy differences of the excited states with respect to their ground state are plotted as a function of the particle number. The number of visible excited states is proportional to the number of energy differences which are smaller than $2|\Delta|$ (red line in Fig. 15). Parameters are $T=0.01 \mathrm{meV}, e V_{b}=0.3 \mathrm{meV},|\Delta|=0.4 \mathrm{meV}, e \Gamma=0.001 \mathrm{meV}$, $b=-0.2 \mathrm{meV}, U=4 \mathrm{meV}$, and $V=2 \mathrm{meV}$.

peaks of the quasiparticle density of states are reflected in the current.

\section{Thermal effects}

We have seen that the shape of the stability diagram can be explained using energy conservation, in complete analogy to the simplest case. In this section we discuss the case for small bias voltages $e V_{b} / 2<|\Delta|$, where current can flow due to thermally excited quasiparticles exclusively. As already observed above, thermally excited quasiparticles do not produce steps in the current-voltage characteristics; rather, they appear as small peaks. This can be used to resolve transitions through excited system states whose energy difference to the ground state is less than $2|\Delta|$. By detuning the gate voltages of the two sites of the DD we can change the level spacing of the systems eigenenergies; hence, the excited states are only observed in a certain detuning range. To analyze transitions through excited system states (cf. Fig. 13), we choose the parallel configuration to rule out the geometrical effect also leading to a decrease of the current for high detuning. If a line corresponding to an excited state disappears for higher detuning $\Delta_{g}$, we conclude that the energy difference to its ground state is larger than $2|\Delta|$. In Fig. 14 we plotted the energy differences of the excited states with respect to their ground state for different values of the detuning $\Delta_{g}$, which are marked as red lines in Fig. 13. Counting the number of levels lying under the red line in Fig. 14 gives information about the number of visible excited lines. For instance, consider the case of $\Delta_{g}=0$ in Fig. 14. Following the red line from small to high $\Sigma_{g}$ in Fig. 13, we cross the 0-1-particle transitions and observe three lines: two corresponding to the ground state, and one line in between corresponds to a transition through the 1-particle excited state. The distance between the leftmost ground state transition line and the excited line determines the level spacing of the 1-particle spectrum (see Fig. 15). In the 2-particle spectrum the energy difference of
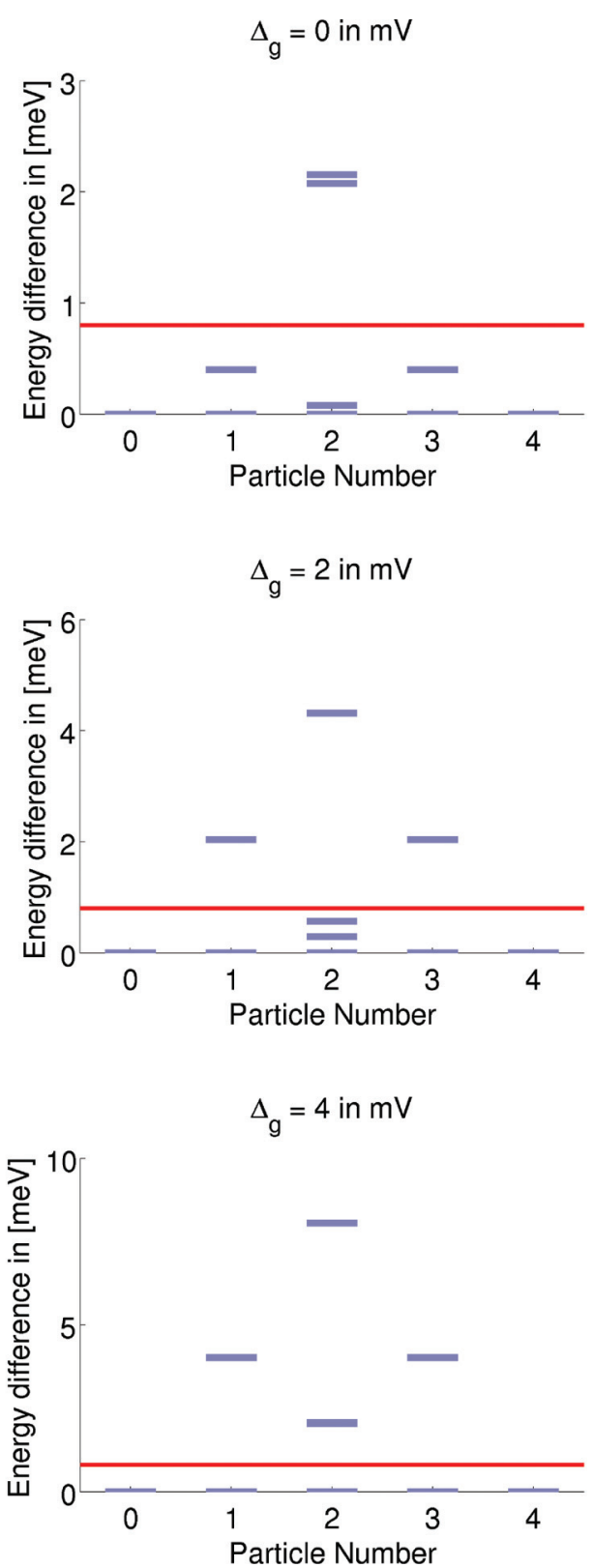

FIG. 14. (Color online) Plot of the energy differences of the excited system states with respect to their ground state as a function of particle number. If the energy difference is smaller than $2|\Delta|$, transitions through these excited states can be seen in the current-voltage characteristics. The threshold of $2|\Delta|$ is marked as a red horizontal line. We depicted the plots for three situations differing in the detuning $\Delta_{g}$. The three cases are marked as horizontal lines in Fig. 13.

one excited state lies under the red line. Hence we should see two lines coming from excited system states, namely, the transition between the 1-particle ground state and the 2-particle excited state, and transitions between the 2-particle ground state and the 1-particle excited state. Along the horizontal cut at $\Delta_{g}=2$ in Fig. 13, excited states can only be observed for the 1-2-particle and the-particle transitions. This is in agreement with Fig. 14, where only in the 2-particle subspace energy differences lie under the threshold of $2|\Delta|$. For higher detuning (e.g., $\Delta_{g}=4$ ), no excited states can be seen, as the 


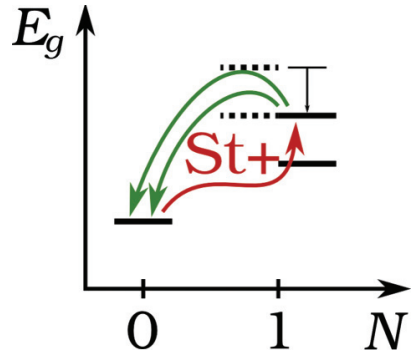

FIG. 15. (Color online) $E_{g}-N$ diagram for the 0-1-particle transition. Transitions between the two 1-particle levels (dashed lines) and the 0-particle ground state are allowed through the thermal St+ transition. Increasing the gate voltage the levels move down in energy (solid lines) and the excited state transition can be observed when the excited level aligns with the $\mathrm{St}+$ transition. Hence, the distance of two neighboring thermal transitions is equal to the level spacing.

detuning increases the level spacing, and all energy differences are larger than $2|\Delta|$ (Fig. 14).

\section{The N-QD-S junction}

We close this paper by investigating a so called N-QD-S hybrid system, where a quantum dot system is coupled to a normal and to a superconducting lead, giving a possible explanation for the subgap features in Ref. 1 . In the experiment of Ref. 1 a carbon nanotube was contacted to two normal conducting leads and to a superconducting finger in between. The differential conductance between the superconducting finger and a normal lead is measured, realizing a N-QD-S hybrid system. It is possible to apply a bias voltage across the entire tube as well as between the superconductor and a normal conducting lead. The stability diagram in Fig. 2(a) in Ref. 1, with no bias applied over the entire tube, reveals the typical Coulomb diamond pattern resulting from quasiparticle tunneling with no subgap features. By applying a bias voltage $V_{\mathrm{SD}}$ over the entire tube, the gap in the stability diagram gets smaller with respect to the unbiased case and conductance lines can be seen in the Coulomb blockade region [cf. Fig. 3(a) of Ref. 1]. The reduction of the gap in the stability diagram is proportional to the applied bias voltage of approximately $e V_{\mathrm{SD}} \approx|\Delta| / 2$, and is related to an effective reduction of the superconducting gap. For a smaller gap quasiparticles can get thermally excited across the gap leading to subgap transport in complete analogy to the S-QD-S case discussed above.

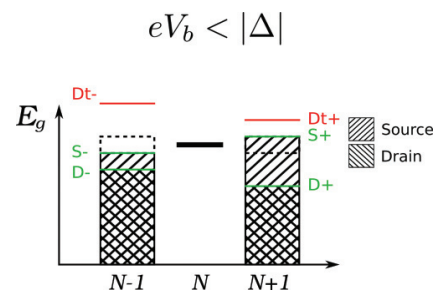

FIG. 16. (Color online) Visualization of the transport conditions for a N-QD-S system with $e V_{b} / 2<|\Delta|$, where the source is a normal and the drain a superconducting lead. They follow from Eqs. (46)(53) by setting $|\Delta|=0$ in the equations corresponding to the source lead.

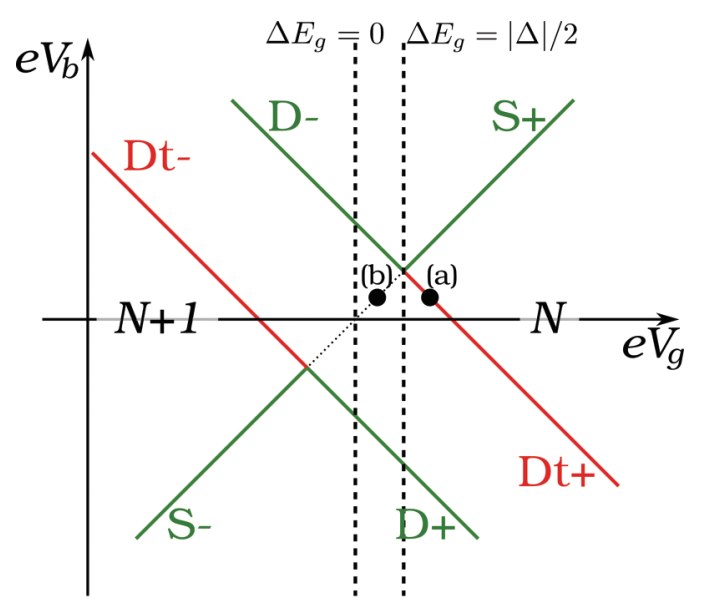

FIG. 17. (Color online) Sketch of the transition line of a QD coupled to a normal conducting (source) and a superconducting lead (drain). The difference to the S-QD-S system is that only the drain lines split due to the superconducting gap; the $\mathrm{S}+$ and $\mathrm{S}-$ lines are described by the same equation. In this case a gap equal to $|\Delta|$ is opening, and the triangles are shifted apart. Thermal lines can be observed only for the drain.

We can model the N-QD-S system by setting $\left|\Delta_{S}\right|=0$ for the normal conducting lead (source) in the master equation; the drain contact remains superconducting $\left|\Delta_{D}\right|=|\Delta|$. Hence, the transport conditions change slightly and can be summarized in the scheme of Fig. 16. In Fig. 17 we schematically sketched the expected transition lines for a N-QD-S hybrid structure. In Fig. 18 we analyzed the two most important cases, marked as points (a) and (b) in Fig. 17. Point (a) shows a paradoxical situation as the particle number of the system seems to be increased only at the drain contact, which would lead to a negative current at positive bias. However, if the two contacts have the same temperature, the thermal broadening of the $\mathrm{S}+$ line gives a small contribution in the transition rates [dashed green arrow in Fig. 18(a)] making the current positive. The situation in Fig. 18(b) shows again the system being in thermal equilibrium with the source contact.

We can see that the lines with negative slope (drain lines) give a finite current in the Coulomb blockade region as observed in Fig. 3(b) in the experiments. Thus, we claim that the subgap features observed in the experiments possibly are transitions involving thermally excited quasiparticles which are allowed due to the reduction of the superconducting gap. This argument is supported by the observation that for (a)

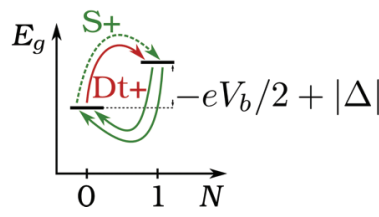

(b)

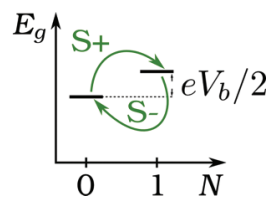

FIG. 18. (Color online) $E_{g}-N$ diagrams corresponding to points (a) and (b) of Fig. 16. (a) We see a positive current in the subgap region, which comes only due to the thermal smearing of the $\mathrm{S}+$ transition. (b) The line connecting the $\mathrm{S}+$ and the $\mathrm{S}-$ transition line in the Coulomb blockade region, the system is in thermal equilibrium with the source contact. 


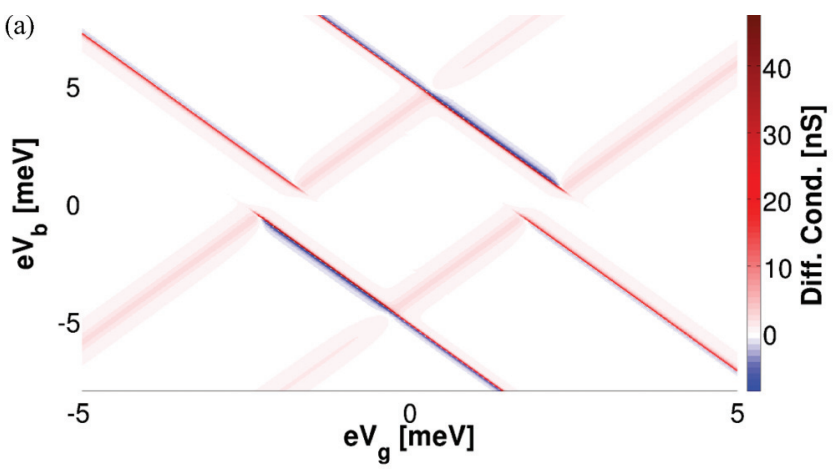

(b)

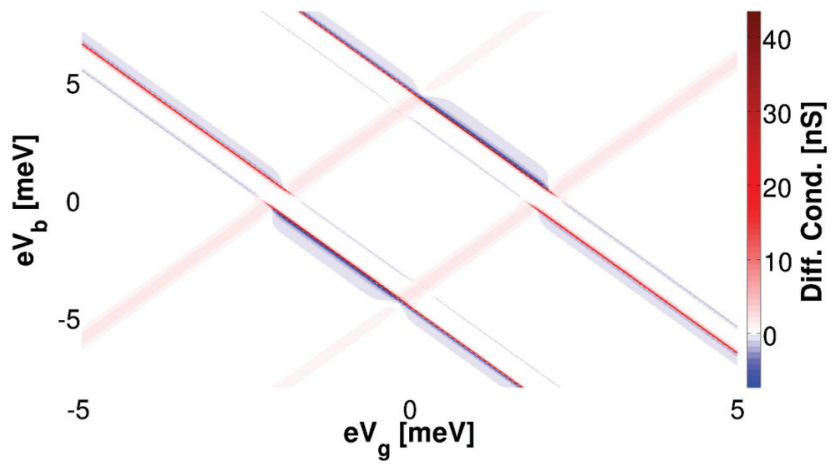

FIG. 19. (Color online) Differential conductance of a SD coupled to a normal conducting (source) and to a superconducting lead (drain) (N-QD-S system). The coupling to the lead is $e \Gamma=0.01 \mathrm{meV}$. (a) Superconducting gap of $|\Delta|=0.6 \mathrm{meV}$ and temperature $k_{B} T=$ $0.1 \mathrm{meV}$. No thermal lines in the subgap region are visible. (b) The same temperature $k_{B} T=0.1 \mathrm{meV}$, but for smaller gap $|\Delta|=0.3 \mathrm{meV}$; quasiparticles get thermally excited across the gap leading to transport in the Coulomb blockade region. Parameters are $U=4 \mathrm{meV}$ and $\epsilon_{d}=-2 \mathrm{meV}$.

diamonds where the gap has the same size as before (edges of the stability diagram), no subgap lines can be observed. In Fig. 19 we show two $d I / d V$ characteristics of a N-QD-S system corresponding to different superconducting gaps with the same temperature $\left(k_{B} T=0.1 \mathrm{meV}\right)$ in both cases. In (b) the superconducting gap $(|\Delta|=0.3 \mathrm{meV})$ is only half of the gap in (a) $\left(k_{B} T=0.6 \mathrm{meV}\right)$. By reducing the gap, the temperature becomes large enough to excite quasiparticles across the gap, leading to conductance peaks in the Coulomb blockade region, as observed in the experiments. However, a more complex modeling of the multiterminal system is required to understand the experiments in all details.

\section{CONCLUSION}

In this work we developed a transport theory for nanostructures coupled to superconducting leads up to second order in the tunneling Hamiltonian. We used the Bogoliubov transformation to describe the electrons in the superconductors as Cooper pairs and Bogoliubov quasiparticle excitations, whereby we modified the Bogoliubov transformation in a number conserving way, ${ }^{25,26}$ introducing Cooper pair creation and annihilation operators explicitly. We showed the predictions of the theory on two examples: the well-known single level quantum dot, and the double quantum dot. The characteristic gap in the Coulomb diamonds, proportional to the superconducting gap, as well as negative differential conductance was observed in both cases. Further, we considered the double quantum dot in serial as well as in parallel configuration (see Fig. 1), coupling the dots to the same as well as to two separate gate electrodes.

We systematically analyzed the stability diagrams, extending the scheme of Ref. 39 for superconducting leads. We found that transport through excited system states occurs even for low bias voltages using thermally excited quasiparticles, leading to zero bias peaks in the conductance. Transitions through excited states can be observed if transitions through the ground state are energetically not allowed, namely, if the distance between the energy levels of the excited state and the ground state is smaller than $2|\Delta|$. This effect can be seen in the the current-voltage characteristics of an independently gated double quantum dot in parallel configuration without tuning parameters of the system, since the level spacing changes with the detuning $\Delta_{g}$ of the gate voltages. Hence the excited states can be seen only in certain detuning windows. Finally, we analyzed the case where a quantum dot is coupled to a normal and a superconducting lead, giving a possible explanation for the subgap features of Ref. 1 in terms of transport involving thermally excited quasiparticles.

We conclude with the observation that thermally excited quasiparticles can lead to a finite current in the Coulomb blockade region. Besides the well-known thermal transitions through the ground states, transitions through excited system states must be taken into account as they are an additional source of zero bias peaks in the conductance. For a better comparison with experiments the theory can be used to investigate more realistic systems such as carbon nanotube quantum double dots. Specifically, the current-voltage spectroscopy in the low bias regime can be used to learn something about the spectrum of the setup. Within our approach it is not possible to capture Josephson current and Andreev reflections as they are higher order processes. Yet, in the weak-coupling regime lowest order quasiparticle transport gives not only the basic structure of the Coulomb diamonds but also the dominant subgap feature, i.e., thermally activated conductance peaks associated to quasiparticle transport. In order to observe the Josephson effect and Andreev reflections, the theory must be extended to higher order perturbation theory. ${ }^{20,22}$

\section{ACKNOWLEDGMENT}

We acknowledge financial support through DFG Program No. SFB631.

\section{APPENDIX A: PROPERTIES OF THE COOPER PAIR OPERATORS}

In the microscopic description of superconductive tunneling it is necessary to know the analytical form of the Cooper pair operators. However, a microscopic discussion of the Cooper pair operators and their influence on the transport properties of the hybrid superconductor-quantum dot junction is rather rare in the literature. In this appendix we show the connection between the Cooper pair operators and ground state of the particle number conserving lead Hamiltonian. Starting from the definition of Eq. (12), we can formally define the 
Cooper pair annihilation operator ${ }^{33}$ as

$$
\hat{S}=\sum_{M=0}^{\infty} \sum_{\left\{n_{k \sigma}\right\}}\left|\left\{n_{k \sigma}\right\}, 2 M\right\rangle\left\langle\left\{n_{k \sigma}\right\}, 2 M+2\right|,
$$

where $\left\{n_{k \sigma}\right\}=\left\{n_{k_{1} \sigma_{1}}, n_{k_{2} \sigma_{2}}, \ldots\right\}$ is a set of quasiparticle occupation numbers. It follows that

$$
\hat{S} \hat{S}^{\dagger}=1,
$$

where we used

$$
1=\sum_{M=0}^{\infty} \sum_{\left\{n_{k \sigma}\right\}}\left|\left\{n_{k \sigma}\right\}, M\right\rangle\left\langle\left\{n_{k \sigma}\right\}, M\right| .
$$

In the full Hilbert space the Cooper pair creation and annihilation operators do not commute:

$$
\left[\hat{S}, \hat{S}^{\dagger}\right]=\hat{\mathcal{P}}_{0},
$$

where $\hat{\mathcal{P}}_{0}$ is the projector to states with zero Cooper pairs:

$$
\hat{\mathcal{P}}_{0}=\sum_{\left\{n_{k \sigma}\right\}}\left|\left\{n_{k \sigma}\right\}, 0\right\rangle\left\langle\left\{n_{k \sigma}\right\}, 0\right| .
$$

Using $\hat{N}\left|\left\{n_{k \sigma}\right\}, M\right\rangle=\left(N_{\left\{n_{k \sigma}\right\}}^{\mathrm{QP}}+M\right)\left|\left\{n_{k \sigma}\right\}, M\right\rangle$, with $N_{\left\{n_{k \sigma}\right\}}^{\mathrm{QP}}$ being the number of quasiparticles in the string $n_{k \sigma}$, one obtains

$$
[\hat{N}, \hat{S}]=-2 \hat{S}, \quad\left[\hat{N}, \hat{S}^{\dagger}\right]=2 \hat{S}^{\dagger}
$$

\section{APPENDIX B: RATES}

\section{Normal rates}

In the stationary limit $\tau \rightarrow \infty$, the normal rates read

$$
\begin{aligned}
\left(\Gamma_{n m m^{\prime} n^{\prime}}^{+}\right)_{\eta}^{N \rightarrow N+1}= & \lim _{\tau \rightarrow \infty}\left(\frac{1}{\hbar}\right)^{2} \sum_{k \sigma \alpha \alpha^{\prime}} t_{\eta \alpha \sigma} t_{\eta \alpha^{\prime} \sigma}^{*}\left\langle n\left|\hat{d}_{\alpha \sigma}\right| m\right\rangle\left\langle m^{\prime}\left|\hat{d}_{\alpha^{\prime} \sigma}^{\dagger}\right| n^{\prime}\right\rangle \int_{0}^{\tau} d t_{2} e^{(i / \hbar) E_{n^{\prime} m^{\prime}} t_{2}} \\
& \times\left[\left|u_{\eta k}\right|^{2} f^{+}\left(E_{\eta k}\right) e^{+(i / \hbar)\left(E_{\eta k}+\mu_{\eta}\right) t_{2}}+\left|v_{\eta k}\right|^{2} f^{-}\left(E_{\eta k}\right) e^{-(i / \hbar)\left(E_{\eta k}-\mu_{\eta}\right) t_{2}}\right], \\
\left(\Gamma_{n m m^{\prime} n^{\prime}}^{+}\right)_{\eta}^{N \rightarrow N-1}= & \lim _{\tau \rightarrow \infty}\left(\frac{1}{\hbar}\right)^{2} \sum_{k \sigma \alpha \alpha^{\prime}} t_{\eta \alpha^{\prime} \sigma} t_{\eta \alpha \sigma}^{*}\left\langle n\left|\hat{d}_{\alpha \sigma}^{\dagger}\right| m\right\rangle\left\langle m^{\prime}\left|\hat{d}_{\alpha^{\prime} \sigma}\right| n^{\prime}\right\rangle \int_{0}^{\tau} d t_{2} e^{(i / \hbar) E_{n^{\prime} m^{\prime} t_{2}}} \\
& \times\left[\left|u_{\eta k}\right|^{2} f^{-}\left(E_{\eta k}\right) e^{-(i / \hbar)\left(E_{\eta k}+\mu_{\eta}\right) t_{2}}+\left|v_{\eta k}\right|^{2} f^{+}\left(E_{\eta k}\right) e^{+(i / \hbar)\left(E_{\eta k}-\mu_{\eta}\right) t_{2}}\right] .
\end{aligned}
$$

In the following we will show how to write the rates in Eqs. (B1) and (B2) in terms of an integral over quasiparticle energies $E_{\eta k}$. Neglecting the lead index $\eta$, the energetic part of Eq. (B1) is proportional to

$$
\left(\Gamma_{n m m^{\prime} n^{\prime}}^{+}\right)^{N \rightarrow N+1} \propto \sum_{k}\left[\left|u_{k}\right|^{2} F_{1}\left(E_{k}\right)+\left|v_{k}\right|^{2} F_{2}\left(E_{k}\right)\right],
$$

where we defined

$$
\begin{aligned}
& F_{1}\left(E_{k}\right)=f^{+}\left(E_{k}\right) e^{(i / \hbar)\left(E_{k}+\omega\right) t_{2}}, \\
& F_{2}\left(E_{k}\right)=f^{-}\left(E_{k}\right) e^{-(i / \hbar)\left(E_{k}-\omega\right) t_{2}},
\end{aligned}
$$

with $\omega=E_{n^{\prime} m^{\prime}}+\mu_{\eta}$. Recalling the definition of $u_{k}$ and $v_{k}$ [cf. Eqs. (10) and (11)], we see that

$$
\left|u_{k}\left(-\xi_{k}\right)\right|=\left|v_{k}\left(\xi_{k}\right)\right| \text {. }
$$

Writing the sum as $\sum_{k} \rightarrow \int_{-\infty}^{\infty} d \xi_{k} \rho_{N}$, and exploiting Eqs. (B5) and (9) we are able to write Eq. (B3) as

$$
\int_{0}^{\infty} d \xi_{k}\left[F_{1}\left(E_{k}\right)+F_{2}\left(E_{k}\right)\right]
$$

Changing the integration variable from $\xi_{k}>0 \rightarrow E_{k}$ we obtain

$$
\int_{|\Delta|}^{\infty} d E D(E)\left[F_{1}(E)+F_{2}(E)\right],
$$

where we defined the superconducting density of states as $D(E)=\rho_{N} \operatorname{Re}\left(\frac{|E|}{\sqrt{E^{2}+|\Delta|^{2}}}\right)$. Due to the definition of the density of states with the real part, we can extend the integral to zero, and use $F_{2}(-E)=F_{1}(E)$ to obtain

$$
\int_{-\infty}^{\infty} d E D(E) F_{1}(E)
$$

\section{Renormalization of the rates}

In the lowest order approximation we find rates which are proportional to the BCS density of states leading to divergences at the gap edges. We can renormalize the rates by introducing a finite lifetime $(\gamma / \hbar)^{-1}$ in the exponents of Eqs. (B1) and (B2). Since we are neglecting coherences the imaginary parts of the rates do not contribute to the dynamics of the system. For example, consider the integral appearing in Eq. (B1):

$$
\begin{gathered}
\operatorname{Re}\left(\int_{-\infty}^{\infty} d E \int_{0}^{\infty} d t_{2} e^{(i / \hbar)(E+\omega+i \gamma) t_{2}} f^{+}(E) D(E)\right) \\
=\int_{-\infty}^{\infty} d E \frac{\hbar \gamma}{(E+\omega)^{2}+\gamma^{2}} f^{+}(E) D(E),
\end{gathered}
$$

where we introduced $\omega=E_{n^{\prime} m^{\prime}}+\mu_{\eta}$. Generalizing the integral for the cases $(N \rightarrow N \pm 1)$ it reads

$$
\hbar \int_{-\infty}^{\infty} d E L(E, \omega) f^{ \pm}(E) D(E)=\hbar \int_{-\infty}^{\infty} d E F(E),
$$


where

$$
L(E, \omega)=\frac{\gamma}{(E+\omega)^{2}+\gamma^{2}}
$$

describes the Lorentzian and $F(E)=L(E, \omega) f^{ \pm}(E) D(E)$. We can solve the integral of Eq. (B10) using residue calculus. To this extent we analyze the singularities of the integrand and the area in which the integrand is analytic. The Lorentzian $L(E, \omega)$ has poles at

$$
E=-\omega \mp i \gamma,
$$

with the corresponding residues:

$$
\operatorname{Res}_{E=-\omega \mp \gamma} L(E)=\frac{ \pm i}{2} .
$$

The poles of the Fermi function $f^{ \pm}(E)$ are purely imaginary and equally distributed along the imaginary axis:

$$
E=\frac{i \pi}{\beta}(2 n+1), \quad n \in \mathbb{Z},
$$

with the residues

$$
\operatorname{Res}_{E=(i \pi / \beta)(2 n+1)} f^{ \pm}(E)=\frac{\mp 1}{\beta} .
$$

The square roots in the BCS density of states $D(E)$ have branch cuts along the real axis. In Fig. 20 we sketched the contour in the complex plane which is slightly shifted away from the real axis with $\epsilon=1 / R$. In the limit $R \rightarrow \infty$ the integral along the

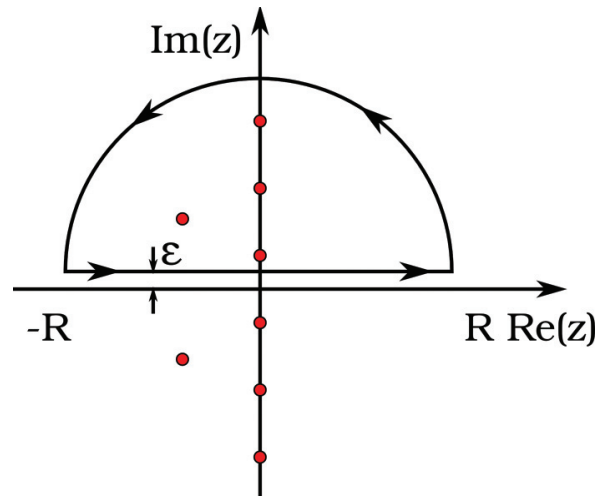

FIG. 20. (Color online) Contour in the complex plane used to integrate Eq. (B10).

semicircle vanishes and we are left with

$$
\lim _{R \rightarrow \infty} \int_{-R}^{R} d x F(x+i \epsilon)=2 \pi i \sum_{\alpha} \operatorname{Res}_{z=\alpha} F(z) .
$$

In the limit $R \rightarrow \infty$ Eq. (B16) is mapped back into the real integral of Eq. (B10), and we find

$$
\begin{aligned}
& \hbar \int_{-\infty}^{\infty} d E L(E) f^{ \pm}(E) D(E) \\
& \quad=\pi \hbar \operatorname{Re}\left[f^{+}(-\omega+i \gamma) D(E-\omega+i \gamma)\right] .
\end{aligned}
$$

*sebastian1.pfaller@physik.uni-r.de

${ }^{1}$ T. Dirks, Y.-F. Chen, N. O. Birge, and N. Mason, Appl. Phys. Lett. 95, 192103 (2009).

${ }^{2}$ M. Buitelaar, W. Belzig, T. Nussbaumer, B. Babić, C. Bruder, and C. Schönenberger, Phys. Rev. Lett. 91, 057005 (2003).

${ }^{3}$ E. Vecino, M. Buitelaar, A. Martín-Rodero, C. Schönenberger, and A. Levy Yeyati, Solid State Commun. 131, 625 (2004).

${ }^{4}$ A. Eichler, M. Weiss, S. Oberholzer, C. Schönenberger, A. Levy Yeyati, J. Cuevas, and A. Martín-Rodero, Phys. Rev. Lett. 99, 126602 (2007).

${ }^{5}$ K. Grove-Rasmussen, H. I. Jørgensen, B. M. Andersen, J. Paaske, T. S. Jespersen, J. Nygård, K. Flensberg, and P. E. Lindelof, Phys. Rev. B 79, 134518 (2009).

${ }^{6}$ L. G. Herrmann, F. Portier, P. Roche, A. Levy Yeyati, T. Kontos, and C. Strunk, Phys. Rev. Lett. 104, 026801 (2010).

${ }^{7}$ J.-D. Pillet, C. H. L. Quay, P. Morfin, C. Bena, A. L. Yeyati, and P. Joyez, Nat. Phys. 6, 965 (2010).

${ }^{8}$ D. Ralph, C. T. Black, and M. Tinkham, Phys. Rev. Lett. 74, 3241 (1995).

${ }^{9}$ J. A. van Dam, Y. V. Nazarov, E. P. A. Bakkers, S. De Franceschi, and L. P. Kouwenhoven, Nature (London) 442, 667 (2006).

${ }^{10}$ Y.-J. Doh, S. De Franceschi, E. P. A. M. Bakkers, and L. P. Kouwenhoven, Nano Lett. 8, 4098 (2008).

${ }^{11}$ L. Hofstetter, S. Csonka, J. Nygard, and C. Schonenberger, Nature (London) 461, 960 (2009).

${ }^{12}$ L. G. Herrmann, P. Burset, W. J. Herrera, F. Portier, P. Roche, C. Strunk, A. L. Yeyati, and T. Kontos, arXiv:1205.1972.
${ }^{13}$ C. B. Winkelmann, N. Roch, W. Wernsdorfer, and V. Bouchiat, Nat. Phys. 5, 876 (2009).

${ }^{14}$ G. Katsaros, P. Spathis, M. Stoffel, F. Fournel, M. Mongillo, V. Bouchiat, F. Lefloch, A. Rastelli, O. G. Schmidt, and S. De Franceschi, Nat. Nanotechnol. 5, 458 (2010).

${ }^{15}$ T. Dirks, T. L. Hughes, S. Lal, B. Uchoa, Y.-F. Chen, C. Chialvo, P. M. Goldbart, and N. Mason, Nat. Phys. 7, 386 (2011).

${ }^{16}$ C. B. Whan and T. P. Orlando, Phys. Rev. B 54, R5255 (1996).

${ }^{17}$ A. Levy Yeyati, J. C. Cuevas, A. Lopez-Davalos, and A. MartinRodero, Phys. Rev. B 55, R6137 (1997).

${ }^{18}$ K. Kang, Phys. Rev. B 57, 11891 (1998).

${ }^{19}$ M. G. Pala, M. Governale, and J. König, New J. Phys. 9, 278 (2007).

${ }^{20}$ M. Governale, M. G. Pala, and J. König, Phys. Rev. B 77, 134513 (2008).

${ }^{21}$ S. De Franceschi, L. Kouwenhoven, C. Schönenberger, and W. Wernsdorfer, Nat. Nanotechnol. 5, 703 (2010).

${ }^{22}$ A. Martín-Rodero and A. L. Yeyati, Adv. Phys. 60, 899 (2011).

${ }^{23}$ B. Andersen, K. Flensberg, V. Koerting, and J. Paaske, Phys. Rev. Lett. 107, 256802 (2011).

${ }^{24}$ K. J. Franke, G. Schulze, and J. I. Pascual, Science 332, 940 (2011).

${ }^{25}$ B. Josephson, Phys. Lett. 1, 251 (1962).

${ }^{26}$ J. Bardeen, Phys. Rev. Lett. 9, 147 (1962).

${ }^{27}$ R. Pariser and R. G. Parr, J. Chem. Phys. 21, 466 (1953).

${ }^{28}$ J. A. Pople, Trans. Faraday Soc. 49, 1375 (1953).

${ }^{29}$ B. R. Bułka and T. Kostyrko, Phys. Rev. B 70, 205333 (2004).

${ }^{30}$ R. Hornberger, S. Koller, G. Begemann, A. Donarini, and M. Grifoni, Phys. Rev. B 77, 245313 (2008). 
${ }^{31}$ N. N. Bogoliubov, Zh. Eksp. Teor. Fiz. 34, 58 (1958) [Sov. Phys. JETP 7, 41 (1958)].

${ }^{32}$ The number of Cooper pairs is not fixed by the chemical potential since $\hat{H}_{G}$ is independent of it. Charging effects would physically determine the total number of electrons and correspondingly the number of Cooper pairs in $|\mathrm{GS}\rangle$.

${ }^{33}$ J. R. Schrieffer, Theory of Superconductivity (W. A. Benjamin, Inc., New York, 1964).

${ }^{34} \mathrm{~V}$. Ambegaokar, in Superconductivity, edited by R. D. Parks, Vol. 1 (Marcel Dekker, Inc., New York, 1969), pp. 259-319.

${ }^{35}$ C. Timm, Phys. Rev. B 83, 115416 (2011).
${ }^{36}$ S. Koller, M. Grifoni, M. Leijnse, and M. R. Wegewijs, Phys. Rev. B 82, 235307 (2010).

${ }^{37}$ K. Blum, Density Matrix Theory and Applications, 2nd ed. (Plenum Press, New York, 1996).

${ }^{38}$ D. S. Kosov, T. Prosen, and B. Žunkovič, J. Phys.: Condens. Matter 25, 075702 (2013).

${ }^{39}$ A. Donarini, G. Begemann, and M. Grifoni, Phys. Rev. B 82, 125451 (2010).

${ }^{40}$ M. G. Schultz and F. von Oppen, Phys. Rev. B 80, 033302 (2009).

${ }^{41}$ W. G. van der Wiel, S. De Franceschi, J. M. Elzerman, T. Fujisawa, S. Tarucha, and L. P. Kouwenhoven, Rev. Mod. Phys. 75, 1 (2002). 